\title{
1 Varicella-Zoster Virus ORF9 Is an Antagonist of the DNA Sensor
} 2 cGAS

3

Jonny Hertzog ${ }^{1}$, Wen Zhou ${ }^{2,3}$, Rachel E. Rigby ${ }^{1}$, Gerissa Fowler ${ }^{1}$, Chiara Cursi ${ }^{1}$, Lise Chauveau ${ }^{1}$, Tamara Davenne ${ }^{1}$, Philip J. Kranzusch ${ }^{2,3,4}$, and Jan Rehwinkel ${ }^{1,{ }^{*}}$

${ }^{1} \mathrm{MRC}$ Human Immunology Unit, MRC Weatherall Institute of Molecular Medicine, Radcliffe Department of Medicine, University of Oxford, Oxford, UK. ${ }^{2}$ Department of Microbiology, Harvard Medical School, Boston, MA, USA.

${ }^{3}$ Department of Cancer Immunology and Virology, Dana-Farber Cancer Institute, Boston, MA, USA.

${ }^{4}$ Parker Institute for Cancer Immunotherapy, Dana-Farber Cancer Institute, Boston, MA, USA.

*correspondence: jan.rehwinkel@imm.ox.ac.uk

\section{SUMMARY}

Varicella-Zoster virus (VZV) causes chickenpox and shingles. Although infection is associated with severe morbidity in some individuals, molecular mechanisms that determine innate immune responses remain poorly defined. We found that the cGAS/STING DNA sensing pathway was required for type I interferon (IFN) induction during VZV infection and that recognition of VZV by cGAS restricted its replication. Viral gene overexpression screening identified the essential VZV tegument protein ORF9 as a cGAS antagonist. Ectopically and virally expressed ORF9 bound to endogenous cGAS. Confocal microscopy revealed co-localisation of cGAS and ORF9, which reduced the type I IFN response to transfected DNA. ORF9 and cGAS also interacted directly in a cell-free system and phase-separated together with DNA. Furthermore, ORF9 inhibited cGAMP production by cGAS. Taken together, we uncovered the importance of the cGAS/STING DNA sensing pathway for VZV recognition and identified a VZV immune antagonist that directly interferes with DNA sensing via cGAS. 


\section{INTRODUCTION}

Varicella-Zoster virus (VZV) is one of nine herpes viruses that infect humans (Arvin and Gilden, 2013). VZV is an alpha-herpesvirus, closely related to herpes simplex virus (HSV) 1 and 2. It has a $125 \mathrm{~kb}$ dsDNA genome, the smallest of the human herpesviruses. The genome includes at least 70 open reading frames (ORFs). Primary infection causes chickenpox (Varicella). Like all human herpes viruses, VZV establishes life-long latency in an infected host and can reactivate as shingles (Zoster). Shingles is a debilitating disease with significant associated morbidity. During both primary infection and reactivation, the virus can gain access to the central nervous system and cause severe complications such as encephalitis and vasculitis (Nagel and Gilden, 2014). Despite introduction of the live-attenuated chickenpox vaccine in the early 1990s, the virus remains highly prevalent worldwide (WHO, 2014).

The type I interferon (IFN) system lies at the forefront of host defence against infectious pathogens and is indispensable for successful control of viral infections ( $\mathrm{McNab}$ et al., 2015). The expression of type I IFNs is induced following pattern recognition receptor (PRR) activation. PRRs are a heterogenous group of proteins that can respond to a diverse array of pathogen-associated molecular patterns (PAMPs) (Brubaker et al., $\underline{2015}$ ). The recognition of viral pathogens relies to a large extent on sensing of nucleic acids (Barrat et al., 2016; Hartmann, 2017). Both endosomal toll-like receptors and dedicated cytosolic sensors are potently activated by viral RNA and DNA. The DNA sensor cyclic GMP-AMP synthase (cGAS) synthesises the second messenger 2'3'cyclic GMP-AMP (hereafter simply cGAMP), a cyclic dinucleotide, upon direct binding to dsDNA (Ablasser and Chen, 2019). Binding of cGAMP to stimulator of IFN genes (STING) results in the activation of the transcription factors IRF3 and NF-KB via the kinases TBK1 and IKKE (Hopfner and Hornung, 2020). IRF3 and NF-KB induce the expression of type I IFNs, type III IFNs, and inflammatory cytokines.

Type I IFNs, including IFNa and IFN $\beta$, are secreted cytokines that act in an autocrine, paracrine, or systemic manner by binding to the type I IFN receptor (IFNAR) (McNab et al., 2015). Canonical IFNAR signalling results in the phosphorylation and heterodimerisation of the transcription factors STAT1 and STAT2. After recruitment of IRF9, this protein complex drives expression of hundreds of genes, termed interferonstimulated genes (ISGs). Among others, ISGs contain genes that encode PRRs, proteins involved in type I IFN induction and signalling, negative and positive feedback regulators, restriction factors acting directly on viruses, and proteins that are involved in adaptive immune responses (Schoggins, 2019).

Despite the fact that VZV is a highly prevalent and important human pathogen, its pathogenesis is still poorly understood. The lack of suitable small animal models that recapitulate primary infection and latency establishment has hindered the molecular characterisation of its life cycle in vivo (Haberthur and Messaoudi, 2013). During its dissemination in the human host, the virus infects a multitude of different cells. Infection of T cells, keratinocytes, neurons, and epithelial cells is indispensable for VZV's life cycle (Zerboni et al., 2014). In addition, immune cells including dendritic cells (DCs), 
77 monocytes, and NK cells are capable of supporting VZV replication in vitro and are

78 potentially relevant for in vivo spread (Abendroth et al., 2001; Campbell et al., 2018;

79 Kennedy et al., 2019; Morrow et al., 2003; Wang et al., 2005). Current evidence suggests

80 that type I IFNs are critical for control of VZV infection. Increased IFNa levels can be

81 detected in the serum of patients with primary VZV infection (Arvin et al., 1986). In

82 addition, type I IFNs limit VZV replication in vitro (Kim et al., 2017; Ku et al., 2016; Shakya

83 et al., 2019; Torigo et al., 2000). However, the events that govern the cell-intrinsic

84 recognition of the virus in the various cell types it infects and induction of the antiviral 85 cytokine response have only begun to be elucidated in vitro. The DNA sensor TLR9 is 86 partly responsible for IFNa secretion after infection of plasmacytoid DCs (Yu et al., 87 2011). In dermal fibroblasts, STING is required for type I and type III IFN production 88 (Kim et al., 2017). An interesting genetic link between DNA sensing via RNA polymerase 89 III and infection of the central nervous system by VZV has been uncovered recently 90 (Carter-Timofte et al., 2018). However, a comprehensive characterisation of the role of 91 DNA sensing during VZV infection is still lacking.

92 In this study we tested which nucleic acid sensors induce type I IFN expression in 93 response to VZV infection. We show that the cGAS - STING - TBK1 - IRF3 signalling 94 axis was responsible for antiviral cytokine expression after VZV infection. We further report the generation of a VZV open reading frame (ORF) expression library and identification of a viral cGAS antagonist. The tegument protein encoded by ORF9 curtailed activation of cGAS and subsequent synthesis of cGAMP. Mechanistically, we show that ORF9 interacted with cGAS and DNA. This resulted in decreased cGAMP and IFN production. We propose a model in which cGAS activation upon VZV infection is limited immediately after viral entry through the tegument protein ORF9. 
101

102

103

104

105

106

107

108

109

110

111

112

113

114

115

116

117

118

119

120

121

122

123

124

125

126

127

128

129

130

131

132

133

134

135

136

137

138

139

140

141

142

143

144

\section{RESULTS}

\section{The Type I IFN Response to VZV Requires the DNA Sensor cGAS}

To identify PRRs that induce type I IFNs in response to VZV infection we used the monocytic cell line THP1. We hypothesised a role of DNA sensors in VZV infection given its identity as a DNA virus and the previously shown role of STING in recognition of VZV (Kim et al., 2017). THP1 cells, unlike many other immortalised cell lines, induce type I and type III IFNs via cGAS in response to DNA (Sun et al., 2013; Wu et al., 2013). Furthermore, THP1 cells are amenable to genome editing and knockout (KO) lines can be used to genetically dissect the role of individual proteins involved in pattern recognition. THP1 cells are permissive for VZV infection and propagation (Nour et al., 2011) and VZV infects primary human monocytes and macrophages in vitro and in vivo (Kennedy et al., 2019; Mainka et al., 1998). In addition to wild-type (WT) THP1 cells, we tested previously described KO lines lacking STING, TBK1, MyD88, or IFNAR2. We further generated cGAS KO, MAVS KO and IRF3 KO cells using CRISPR/Cas9 technology (see Materials and Methods and Figures S1 and S2). All THP1 KO cells were validated by immunoblotting for the absence of protein and functionally by stimulation with DNA, RNA and type I IFN (Figures S1, S2). These cells contained a secreted luciferase reporter construct under control of an IRF3-responsive promotor. Upon treatment with PMA, THP1 cells adopt a macrophage-like, adherent and highly responsive phenotype. Given the difficulties of working with cell-free VZV (Caunt and Taylor-Robinson, 2009; Chen et al., 2004), we used co-culture with VZV-infected (+VZV) MeWo cells to infect PMA-treated THP1 cells; co-culture with uninfected MeWo cells served as a control (Figure S3A). MeWo cells are a melanoma cell line that is wellestablished for VZV propagation. After 48 hours of co-culture, cells were harvested for RT-qPCR and immunoblotting. For all experiments, uninfected MeWo cells were additionally used as target cells. MeWo cells do not induce type I IFNs in response to VZV (Figure S3B).

To investigate whether THP1 cells induce type IIFNs in response to VZV we analysed mRNA expression levels of IFNB1 (encodes IFN $\beta$ ) and IFI44, an ISG. In WT THP1 cells, both transcripts were robustly induced after VZV infection (Figure S3B). Similar results were obtained using MyD88 KO and MAVS KO cells. In contrast, no transcriptional upregulation of IFNB1 or IFI44 was observed in THP1 cells lacking cGAS, STING, TBK1, IRF3, or IFNAR2. Moreover, immunoblot analysis showed that the transcription factors STAT1 and STAT2 were only phosphorylated in WT, MyD88 $\mathrm{KO}$, and MAVS KO cells (Figure S3C). No p-STAT1 and p-STAT2 signals were observed in cells lacking cGAS, STING, TBK1, IRF3, or IFNAR2. This indicated that only WT, MyD88 KO and MAVS KO cells secreted type I IFNs in response to VZV infection. Consistently, STAT1 and RIG-I, which are both encoded by ISGs, were upregulated at protein level only in the cells showing STAT1/2 activation (Figure S3C). Importantly, we could not observe phosphorylation of STAT1/2 or increased abundance of STAT1 and RIG-I in infected MeWo cells. Western blotting with antibodies against VZV-glycoprotein E (gE)/glycoprotein I (gl) and VZV ORF62 confirmed that all cell lines became infected (Figure S3C). Determination of CXCL10 
(IP-10) levels in co-culture supernatants confirmed the findings of our RT-qPCR and immunoblot analyses. WT, MyD88 KO, and MAVS KO THP1 cells produced low levels of CXCL10 at baseline and these were increased after infection with VZV (Figure S3D). No CXCL10 was detected in supernatants from uninfected cells and in samples from infected cGAS KO, STING KO, TBK1 KO, and IFNAR2 KO THP1 cells; similarly, MeWo cells did not secrete CXCL10. We could detect low levels of CXCL10 in supernatants from IRF3 KO cells but there was no increase above baseline after infection. Collectively these results suggest that in THP1 cells the type I IFN response to VZV infection required the DNA sensor cGAS and the STING - TBK1 - IRF3 signalling axis. It is therefore likely that dsDNA is the PAMP recognised in VZV-infected cells.

To dissect the role of the DNA sensor CGAS in recognition of VZV further we developed a transwell-based infection system (Figure 1A). In this setup, infected MeWo cells are first seeded on the bottom side of the transwell membrane. After adherence, THP1 target cells are seeded on the opposite side of the membrane. The membrane contains $1 \mu \mathrm{m}$ pores through which cell-cell contacts can be established and VZV can spread. Importantly, the inoculum and target cells do not mix, and a homogenous target cell population can be harvested for analysis. We anticipate that this new infection protocol (see Methods for details) will be widely applicable to many VZV research projects. WT, cGAS KO, and MAVS KO THP1 (Figure 1B) cells were infected for 24 and 48 hours using this transwell assay. Cell-surface staining for the VZV-gE/gl complex and flow cytometry analysis (Mo et al., 2003) revealed significantly higher levels of infection in cGAS KO cells compared to WT and MAVS KO cells at both time points (Figures $1 \mathrm{C}$ and S4). We further found increased expression of immediate early (IE), early (E), and late (L) viral gene products in cells lacking cGAS (Figure 1D). In line with our previous results, THP1 cells failed to upregulate IFNB1 and ISG expression after VZV infection in the absence of cGAS (Figure 1E). In WT and MAVS KO cells, VZV infection robustly induced secretion of the IRF3-controlled luciferase reporter and CXCL10 (Figure 1F,G). This response was undetectable in cGAS KO cells. These results confirm our previous observations from the co-culture system and establish that the type I IFN response to VZV infection in THP1 cells was mediated by the DNA sensor cGAS. Significantly more cells became infected with VZV in the absence of cGAS, indicating that recognition by cGAS was required for restriction of VZV infection.

\section{A VZV ORF Expression Library}

Type I IFNs inhibit VZV infection (Kim et al., 2017; Ku et al., 2016). VZV, like many other viruses, employs immune evasion strategies that target the type I IFN system. For example, both ORF61 and ORF62 limit IRF3 activation through distinct mechanisms (Sen et al., 2010; Zhu et al., 2011). In light of our finding that cGAS was crucial for type I IFN induction in response to VZV, we hypothesised that the virus expresses a direct antagonist of cGAS and/or STING. Indeed, other large DNA viruses often encode multiple antagonists of the same innate immune pathway ( Smith et al., 2018; Stempel et al., 2019). In order to test the role of individual viral gene products in immune evasion 
we generated an expression library for all canonical VZV ORFs. All coding sequences were PCR-amplified and cloned into a gateway entry vector. Using recombination, these sequences were then shuttled into a lentiviral vector (pLenti6.3/TO/V5). This vector allows expression with a C-terminal V5 epitope tag either after transient transfection or via lentiviral transduction. To validate these constructs, we transiently transfected HEK293T cells and analysed expression of VZV proteins by immunoblot using an antibody against the V5 tag (Figure S5). 59 of 72 constructs (82\%) were expressed and bands at the expected molecular weights were detected. An additional five constructs were expressed but not at the expected size, and eight were not expressed at detectable levels. This VZV ORF library is a resource for the scientific community and is available to all interested scientists.

\section{VZV ORF9 is an Antagonist of DNA sensing}

To investigate whether VZV ORFs block cGAS/STING activation, we utilised a luciferase-based screening platform in HEK293T cells. In brief, a plasmid expressing Firefly luciferase under IFNB1 promotor control and pRL-TK, which constitutively expresses Renilla luciferase, were transiently transfected. HEK293T cells do not express CGAS and STING naturally. We therefore reconstituted human CGAS and human STING by transient transfection, which results in activation of the IFNB1 promotor and firefly luciferase expression. Lastly, individual viral ORFs (or controls) were co-expressed. Firefly luciferase expression was normalised to Renilla luciferase expression, and we calculated for each ORF a luciferase fold change to an empty vector control condition without CGAS and STING expression constructs. The mean and standard deviation of all data points was then used to calculate Z-values, which represent the number of standard deviations an individual data point is diverging from the mean. We used these Z-scores to rank ORFs in their ability to block IFN activation downstream of cGAS/STING (Figure 2A). KSHV ORF52, a previously described cGAS-antagonist (Wu et al., 2015), and the L protein of EMCV, a previously described IRF3-antagonist (Freundt et al., 2018), served as positive controls. As expected, we found these with the lowest ranks (i.e. smallest fold change) in our assay and both potently blocked firefly luciferase induction. In addition, we identified a number of VZV ORFs that showed similar behaviour. The two aforementioned IRF3 antagonists expressed by VZV, ORF61 and ORF62, were among them, which further validated our approach.

We performed this screening experiment three times; Figure 2B displays the results as a heatmap. We identified a number of ORFs that reproducibly ranked very low. To identify VZV proteins that specifically block cGAS and/or STING, and not downstream signalling proteins such as IRF3 that are also activated by other PRRs, we compared selected hits from the primary screen in their ability to block reporter activation by overexpression of cGAS/STING or RIG-I-CARD (data not shown). RIG-I-CARD is a constitutively active variant of RIG-I that activates the IFNB1 promotor via MAVS. A direct cGAS/STING antagonist is therefore unable to block this stimulation. We identified the protein encoded by ORF9 of VZV to selectively block reporter activation by cGAS/STING but not RIG-I-CARD when compared to empty vector (Figure $2 \mathrm{C}$ ). 
We then aimed to verify that VZV ORF9 antagonises activation of cGAS by dsDNA in an endogenous setting. THP1 monocytes were stably transduced with ORF9-V5 or GFP-V5 as a negative control (Figure 2D). We stimulated cGAS in these cells by transfection of increasing doses of dsDNA and measured expression levels of IFNB1 mRNA and IFNL1 mRNA (encoding a type III IFN) by RT-qPCR (Figure 2E). As expected, THP1 cells expressing GFP showed a dose-dependent increase in expression of both transcripts. In contrast, cells expressing ORF9 did not respond to low doses of DNA. At higher doses, their response was attenuated when compared to GFP-expressing cells. Collectively, these data revealed that the VZV ORF9 protein prevented cGAS/STING activation.

\section{ORF9 Interacts With cGAS}

We hypothesised that VZV ORF9 exerts its antagonistic function by directly interacting with either cGAS or STING. To test this, HEK293T cells were transiently transfected with expression constructs for cGAS-FLAG, STING-HA, and ORF9-V5 and protein interaction was analysed by immunoprecipitation (IP). We used antibodies against the epitope tags and analysed IP fractions by immunoblotting (Figure 3A). All ectopically expressed proteins were precipitated efficiently, and an IgG isotype control antibody did not precipitate any of the proteins tested. Interestingly, ORF9 was detected in the bound fraction after cGAS IP. The reverse IP confirmed this result: we found cGAS in the ORF9 IP. In contrast, ORF9 did not interact with STING in this assay (Figure 3A). To verify that this interaction occurred with endogenous cGAS, we used THP1 cells stably transduced to express FLAG-ORF9, which was precipitated from cell lysates with $\alpha-F L A G$ antibody. Indeed, endogenous cGAS was present in the IP fraction; IP of FLAG-GFP served as a negative control (Figure 3B).

Next, we asked whether ORF9 expressed from its endogenous promotor during viral infection had the ability to interact with cGAS. We used a recombinant VZV that expressed C-terminally V5-tagged ORF9 from its endogenous genomic locus. We infected THP1 cells and HaCaT cells (a keratinocyte cell line that expresses cGAS) with WT VZV or VZVORF9-V5 and performed $\alpha$-V5 IP (Figure 3C). In both THP1 and HaCaT cells infected with VZVORF9-V5, endogenous cGAS co-precipitated with ORF9. These data showed that endogenous ORF9 protein expressed by the virus in infected cells interacted with cGAS.

To investigate whether the two proteins interact in cells, we overexpressed tagged ORF9 and cGAS in HEK293T cells and performed immunofluorescence analysis (Figure 3D). Expression of ORF9 alone resulted in cytoplasmic staining. Similarly, cGAS was detected in the cytoplasm. We further observed cGAS foci co-localising with extranuclear DNA. Extranuclear DNA foci, sometimes in the form of micronuclei, can be observed in some cancer cells and have been shown to bind cGAS (Harding et al., 2017; Hu et al., 2019; Mackenzie et al., 2017). Interestingly, when ORF9 and cGAS were expressed together, both proteins co-localised in DAPI-positive, extranuclear regions. This indicated that ORF9 interacted with cGAS in cells and localised together with cGAS in DNA-positive areas. 
In order to biochemically characterise the interaction between ORF9 and cGAS in more detail, we performed experiments in a cell-free system. We expressed cGAS, cGASHA, FLAG-ORF9, and FLAG-GFP in E. coli and performed single-step purification (Figure S6A). First, we tested whether ORF9 and cGAS interacted directly using the far western protocol (Figure 3E). FLAG-ORF9 or FLAG-GFP protein were separated on a denaturing SDS-PAGE gel and transferred to a membrane. The proteins on the membrane were then re-natured and incubated with recombinant cGAS as a probe. Binding of cGAS to proteins on the membrane was then tested using $\alpha$-cGAS antibody. Indeed, we found that probing for cGAS resulted in a signal at the size of ORF9 (Figure 3F). Importantly, cGAS did not bind to GFP.

We then tested interaction of the two recombinant proteins using immunoprecipitation. FLAG-ORF9 or FLAG-GFP were incubated with cGAS-HA in the test tube. The proteins were then precipitated using antibodies against the epitope tags. Immunoblot analysis of the IP fractions showed that CGAS was co-immunoprecipitated with ORF9 but not GFP (Figure 3G). The reverse IP of cGAS resulted in binding of both ORF9 and GFP; however, the signal was stronger for ORF9. Taken together, these data indicate that VZV ORF9 and cGAS interacted without the requirement for another cellular or viral protein.

To characterise the interaction of ORF9 and cGAS mechanistically, we constructed ORF9 truncation mutants (Figure 4A). We tested their ability to interact with cGAS by co-IP after overexpression in HEK293T cells (Figure 4B). Consistent with our earlier observation, full-length ORF9 co-immunoprecipitated cGAS. The C-terminal half of ORF9 (construct II) behaved the same, while the N-terminal half (construct I) failed to interact with cGAS. ORF9 constructs III, IV, and V also pulled down cGAS. All ORF9 constructs that interacted with cGAS shared amino acids (AA) 151 to 240 . However, IP of this region in isolation (ORF9 ${ }^{151-240}$, construct VI) did not co-precipitate cGAS (Figure $4 \mathrm{~B}$ ). Extension of this construct at both ends by about 10 amino acids to generate construct VIII (AA 141 to 249 ) restored robust interaction with cGAS (Figure 4B).

\section{ORF9 Binds DNA}

To gain insight into possible structural features of ORF9 in this region we used the PSIPRED 4.0 algorithm to predict its secondary structure based on the AA sequence (Buchan and Jones, 2019). This analysis predicted a two helix - sheet - helix motif in the C-terminal region of ORF9 (Figure 4C). We obtained a similar secondary structure prediction for the C-terminal half of VP22, the HSV-1 homologue of VZV ORF9. A crystal structure is available for this region of VP22 ( presence of the predicted two helix - sheet - helix motif. Hew et al. further identified a structural similarity of VP22 with the unrelated ORF52 protein of murine herpesvirus 68 (MHV68). MHV68 is closely related to human herpesvirus 8, also known as Kaposi sarcoma associated herpesvirus (KSHV). KSHV ORF52 has been identified as a cGAS antagonist and has DNA-binding properties (Wu et al., 2015). This led us to hypothesise that ORF9 interacts with DNA. To test this, we incubated biotinylated VACV-70mer dsDNA with recombinant ORF9; recombinant cGAS and GFP served as positive and 
negative controls, respectively. The VACV-70mer is a well-established immunostimulatory dsDNA that binds cGAS (Almine et al., 2017; Lum et al., 2018; Unterholzner et al., 2010). The DNA was then precipitated using streptavidin beads and the presence of bound proteins was analysed by immunoblotting (Figures 4D and $\mathrm{S} 6 \mathrm{~A}$ ). In the absence of DNA, none of the proteins were precipitated. As expected, cGAS bound to DNA. Interestingly, ORF9 was also pulled down by DNA, both alone and in the presence of cGAS. GFP did not bind DNA under any conditions. We further overexpressed either FLAG-ORF9 or FLAG-GFP in HEK293T cells and performed a similar precipitation experiment after spiking the lysates from these cells with VACV70mer-Biotin (Figure 4E). As expected, ORF9, but not GFP, could be pulled down with DNA. To characterise the interaction of ORF9 and DNA in more detail, we performed agarose gel electromobility shift assays (EMSA) (Figures 4F and S6B). Recombinant ORF9 protein impaired the mobility of VACV70mer dsDNA, indicating the formation of ORF9-DNA complexes. These increased in size with higher doses of ORF9, suggesting multivalent protein-protein/protein-DNA interactions. We performed similar experiments with full-length human cGAS protein. As previously described (Zhou et al., 2018), hcGAS and DNA form high molecular weight (HMW) complexes which are unable to migrate out of the gel pocket. Next, we tested incubation of VACV70mer and cGAS with increasing doses of ORF9 protein. Addition of ORF9 to cGAS and DNA increased the size of protein/DNA complexes observed at this concentration of cGAS alone. Taken together, these data show that ORF9 interacted with both DNA and cGAS, without displacing cGAS from DNA.

\section{ORF9 Phase Separates With DNA}

Liquid-liquid phase separation contributes to cGAS activation by dsDNA (Du and Chen, 2018; Xie et al., 2019; Zhou et al., 2021). This is driven by multivalent interactions between cGAS and DNA. In light of our results that ORF9 bound cGAS and DNA, we investigated the effect of ORF9 on cGAS-DNA phase separation. As reported previously, we observed droplet formation by human cGAS and labelled dsDNA, which was sensitive to increasing salt concentration $>250 \mathrm{mM}$ (Figures $5 \mathrm{~A}$ and S6C). Similarly, ORF9 and labelled dsDNA formed liquid droplets in the absence of cGAS (Figures 5A and S6C). ORF9-DNA droplets were smaller than cGAS-DNA droplets, which may indicate a lower propensity of ORF9 to phase separate with DNA compared to cGAS. A C-terminally truncated version of ORF9 (ORF9-N, AA 1-244) had much reduced ability to form liquid droplets (Figures $5 \mathrm{~A}$ and $\mathrm{S6C}$ ). Next, we formed cGASDNA droplets in the presence of ORF9 and observed that ORF9 had little effect on droplet formation by cGAS and DNA (Figure 5B). Therefore, ORF9 did not antagonise phase separation of cGAS and dsDNA, suggesting another mechanism of cGAS inhibition.

\section{VZV ORF9 Inhibits cGAMP Synthesis}

We next sought to establish the importance of DNA binding for ORF9's function as a cGAS antagonist. Alignment of the ORF9 with related herpesvirus proteins (Figure 4C) revealed multiple conserved, positively-charged AA residues (Figure 6A). This 
included ORF9 K178A/R179A and ORF9 R186/R187. The latter aligned with KSHV ORF52 R68/K69 that are required for DNA binding (Wu et al., 2015). We therefore generated three ORF9 mutants: K178A/R179A (construct A), R186A/R187A (construct $B$ ), and a double mutant (DM), in which all four residues were mutated to alanine. The mutation sites $A$ and $B$ are located at the beginning and in the middle, respectively, of the first alpha helix in the predicted two helix - sheet - helix of ORF9 (Figure 6B). In the HSV-1 VP22 crystal structure, the corresponding helix forms part of a large, positively charged groove, consistent with a possible role in DNA-binding ( test the effect of these mutations experimentally, we performed DNA pull-down experiments. As observed before (Figure 4E), WT ORF9 robustly precipitated with DNA (Figure 6C). In contrast, both the $A$ and $B$ mutant were impaired in their ability to bind DNA, with a stronger effect for the B-site. The double mutant did not detectably interact with DNA. Next, we investigated whether the mutant ORF9 proteins interacted with cGAS (Figure 6D). Mutation of either the A or B site alone had no effect on cGAS binding but ORF9-DM showed attenuated cGAS binding.

To test the ORF9-DM protein functionally, we generated THP1 cell lines that stably overexpressed GFP, ORF9, or ORF9-DM and stimulated these cells with increasing doses of dsDNA (Figure 6E). Compared to GFP-expressing cells, we found equally reduced IFNB1 induction in cells expressing either WT or mutant ORF9. These results indicate that the interaction of ORF9 and cGAS was partially dependent on ORF9's ability to bind DNA. Intriguingly, however, DNA-binding by ORF9 was not required for its ability to inhibit DNA sensing.

Lastly, we used a cell-free cGAMP synthesis assay (Kranzusch et al., 2013) to test whether ORF9 inhibits cGAS enzymatic activity directly. For this, recombinant human cGAS was incubated with radioactively labelled ATP, GTP, DNA, and recombinant ORF9 or bovine serum albumin (BSA) as control. The radioactively-labelled 2'3'cGAMP produced in these reactions was visualised by thin-layer chromatography and phosphorimaging (Figure 6F and S6B). While addition of BSA did not affect cGAMP production at any dose, ORF9 inhibited cGAS activity in a dose-dependent manner (Figures 6F and 6G). At the doses tested, ORF9-DM was equally potent in inhibiting cGAS compared to WT ORF9.

In sum, these results showed that ORF9 directly inhibited the enzymatic activity of cGAS by a mechanism that did not require DNA binding by ORF9. 


\section{DISCUSSION}

398

399

400

401

402

403

404

405

406

407

408

409

410

411

412

413

414

415

416

417

418

419

420

421

422

423

424

425

426

427

428

429

430

431

432

433

434

435

436

437

438

439

440

VZV is a highly prevalent human virus, yet little is known about its host-pathogen interactions and innate immunity. Here we report that cGAS and its downstream signalling pathway consisting of STING, TBK1 and IRF3 were required for type I IFN induction after VZV infection. An earlier study using RNA interference had implicated STING in innate recognition of VZV (Kim et al., 2017). Our results confirm this observation by complete genetic ablation, and - importantly - identify cGAS as the DNA sensor for VZV. It is possible that innate sensing pathways that detect VZV differ between cell types. Indeed, a study using inhibitory oligonucleotides found that plasmacytoid DCs produce type I IFN in a partially TLR9-dependent manner (Yu et al., 2011). In contrast, we found that the essential TLR9 adaptor protein MyD88 was dispensable for type I IFN induction in VZV-infected THP1 cells. It will be interesting to characterise innate sensing pathways in different cell types relevant to in vivo infection, including neuronal cells, T cells, and skin cells. This is important because the viral life cycle and effects of viral replication on host cells can differ between cell types (Zerboni et al., 2014). For example, VZV shows cytopathic effects in fibroblast and some immune cells but does not induce cell death in neurons (Gerada et al., 2020). Future investigation should therefore not only address the role of PRRs in induction of cytokines such as type I IFNs but also in induction of cell death (Maelfait et al., 2020).

Our identification of cGAS as a sensor of VZV infection implicates recognition of an immunostimulatory DNA in infected cells. Studies of herpesvirus entry suggest that the viral DNA remains within the viral capsid during nuclear targeting and may therefore be unavailable for binding to the cytosolic pool of cGAS (Radtke et al., 2006). Single-cell analysis of HSV-1 infection showed that only cells undergoing abortive infection respond by production of type I IFNs (Drayman et al., 2019). It is therefore possible that viral particles with defective capsids are responsible for the type I IFN response observed in bulk populations of cells. Furthermore, cellular restriction mechanisms may make viral DNA from capsids accessible for cGAS binding. Indeed, degradation of herpesviral capsids via the ubiquitin - proteasome system has been suggested to release viral DNA for recognition (Horan et al., 2013). Alternatively, cellular DNA may induce type I IFN. Indeed, infections with multiple different viruses result in the accumulation of extranuclear DNA and in mitochondrial damage (Ablasser and Chen, 2019). Host DNA has been shown to stimulate cGAS in the context of HSV-1 infection (West et al., 2015). Whether viral and/or cellular DNA species activate cGAS in VZVinfected cells should be tested in the future by deep sequencing of cGAS-bound DNA. However, this approach has thus far been hampered by the lack of suitable antibodies for specific cGAS IP.

We further describe the identification of ORF9 as a cGAS antagonist. ORF9 interacted with cGAS in a variety of assays, and inhibited cGAMP production and DNA-triggered type I IFN induction. ORF9 is a tegument protein, making it an attractive candidate for antagonising innate immunity. Tegument proteins are contained within viral particles and are delivered into cells at the same time as viral DNA. ORF9 may therefore limit DNA sensing during early infection before viral gene expression. At first glance, our 
findings that cGAS recognises VZV infection and that ORF9 inhibits cGAS activation might appear contradictory. However, viral immune evasion mechanisms typically limit but not entirely suppress innate immune responses. This notion is supported by the observation that in some cases multiple viral antagonists target the same host defence pathway (Smith et al., 2018; Stempel et al., 2019). In addition, cGAS activation may occur partly in cells infected with defective virions (Drayman et al., 2019) that are likely to contain and/or express reduced amounts of ORF9. What is the molecular mechanism by which ORF9 inhibits cGAS? Formation of multimeric complexes and higher order structures of cGAS and DNA facilitate cGAS activation (Andreeva et al., 2017; Du and Chen, 2018; Li et al., 2013; Zhang et al., 2014; Zhou et al., 2018). We found that ORF9 binds DNA and phase separated with DNA. We therefore speculated that ORF9 might disrupt cGAS-DNA oligomers. However, further gel shift and phase separation experiments falsified this hypothesis; in contrast, ORF9 might actually facilitate formation of high-molecular weight complexes containing cGAS and DNA. Moreover, an ORF9 mutant with abrogated DNA binding retained the ability to bind cGAS and to inhibit cGAMP production in vitro and IFN $\beta$ induction in cellulo. Although we cannot exclude that this mutant retained weak DNA-binding below the sensitivity of our assays, we prefer a model in which direct protein-protein interactions between ORF9 and CGAS are sufficient for inhibition of cGAMP production. DNA-binding of ORF9 may have unrelated functions and/or may facilitate cGAS interaction and inhibition in specific settings. Future experiments are required to decipher in detail the relationships between ORF9, cGAS, DNA-binding, and cGAMP production. This could include testing DNA length requirements as cGAS oligomer formation is dependent on DNA length (Andreeva et al., 2017; Li et al., 2013; Luecke et al., 2017; Zhou et al., 2018).

467

ORF9 is essential for viral replication and is a member of the $\alpha$-herpesvirus UL49 gene family (Che et al., 2008; Tischer et al., 2007). With its closest relative, HSV-1 VP22, it shares 36\% AA sequence similarity ( $\mathrm{Hew}$ et al., 2015). ORF9 has well-established roles in VZV nuclear egress and secondary envelopment (Che et al., 2013; Lebrun et al., 2018; Riva et al., 2013; Riva et al., 2015). Mutational analyses have attributed these functions to AAs or regions either in the $\mathrm{N}$-terminal half or the extreme $\mathrm{C}$-terminus of the protein, whilst we describe a central region (AA151-240) to be required for the interaction of ORF9 with cGAS. A crystal structure is available for the core domain of HSV-1 VP22 that is homologous to this region of ORF9 ( folds into a two helix - sheet - helix motif. Hew et al. further describe structural similarity between HSV1 VP22 and the unrelated MHV68 ORF52 (Hew et al., 2015). MHV68 ORF52 is the homologue of KSHV ORF52. Secondary structure prediction and examination of the published crystal structures revealed that VZV ORF9, HSV1 VP22, KSHV ORF52, and MHV68 ORF52 all potentially share a two helix - sheet structural feature (Figure 4C). Interestingly, both HSV-1 VP22 and KSHV ORF52 have been described previously to inhibit cGAS activation (Huang et al., 2018; Wu et al., 2015). Antagonism of cGAS by KSHV ORF52 requires its DNA binding properties (Wu et al., 2015). In contrast, we found that ORF9's DNA-binding ability was not required for inhibition of cGAS. This indicates that while structural similarity between the 
485 aforementioned proteins could confer cGAS inhibitory properties, the precise 486 molecular mechanisms might differ. In addition, VZV ORF9 and KSHV ORF52 are 487 essential viral gene products while HSV-1 VP22 is not required for viral replication 488 (Huang et al., 2018; Li et al., 2016). Future experiment will be required to shed light on 489 the precise relationship between these viral proteins.

490 Collectively, our observations lead us to put forward a model in which distantly related 491 herpesviruses have retained within unrelated proteins a structural feature that confers 492 cGAS antagonist properties. Alpha, beta, and gamma-herpesviruses have been 493 estimated to have evolutionarily diverged hundreds of millions of years ago (Brito and 494 Pinney, 2018; Davison, 2002; McGeoch et al., 1995). Anemone species that have diverged 495 from humans more than 500 million years ago harbour cGAS-like enzymes (Kranzusch 496 et al., 2015). This indicates that a common ancestral organism expressed such proteins. 497 It further opens up the possibility that ancient herpes viruses evolved the relevant 498 evasion strategies. We hypothesise that antagonism of cGAS by herpesviruses 499 constitutes an ancient molecular mechanism that evolved long before the advent of 500 antiviral cytokines and IRF3 during evolution. 
501

502

503

504

505

506

507

508

509

510

511

512

513

514

515

516

517

518

519

520

521

522

523

524

525

526

527

528

529

530

531

\section{AUTHOR CONTRIBUTIONS}

$\mathrm{JH}$, RER, and JR conceived the study. JH, WZ, RER, PJK, and JR designed experiments and analysed data. JH, WZ, GF, CC, LC, and TD performed experiments. $\mathrm{JH}, \mathrm{WZ}$, and RER developed methodology. JH and JR wrote the manuscript with help from all authors. All authors read and approved the final manuscript.

\section{ACKNOWLEDGMENTS}

The authors thank J Cohen, C Sadzot and S Paludan for VZV stocks, antibodies and cell lines, and P Hublitz and Z Holloway (MRC WIMM Genome Engineering Facility) for their help with CRISPR plasmid generation. The authors further thank $C$ Reis $e$ Sousa, F Zhu, G Ogg, L Unterholzner, A Pichlmair, G Towers, T Fujita, J Haas, and M Reijns for reagents and advice. The authors thank M Brinkmann, J McKeating, and members of the Rehwinkel lab for critical discussion.

\section{FUNDING}

This work was funded by the UK Medical Research Council [MRC core funding of the MRC Human Immunology Unit], the Wellcome Trust [grant number 100954], and the Concern Foundation. $\mathrm{JH}$ was supported by the European Commission under the Horizon2020 program H2020 MSCA-ITN GA 675278 EDGE. WZ was supported through a Charles A. King Trust Postdoctoral fellowship. The MRC WIMM Genome Engineering Facility is supported by grants from the MRC/MHU (MC_UU_12009), the John Fell Fund (123/737) and by MRC WIMM Strategic Alliance awards G0902418 and MC_UU_12025. The funders had no role in study design, data collection and analysis, decision to publish, or preparation of the manuscript.

\section{CONFLICT OF INTEREST}

The authors declare no conflict of interest. 
A

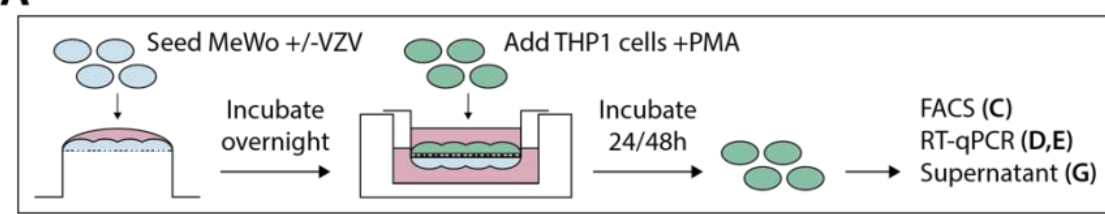

C

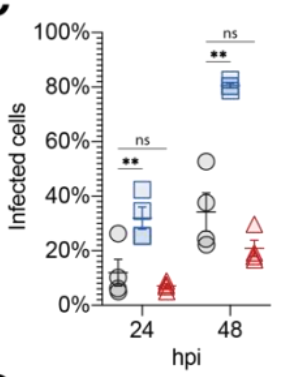

D

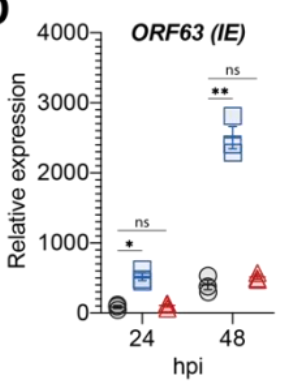

E

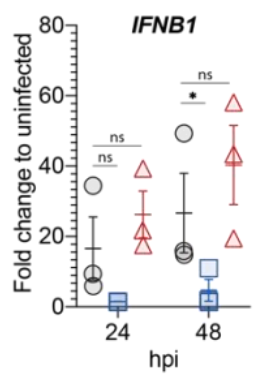

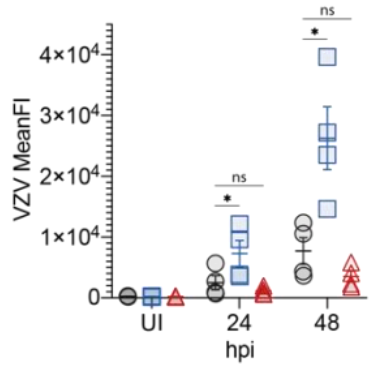
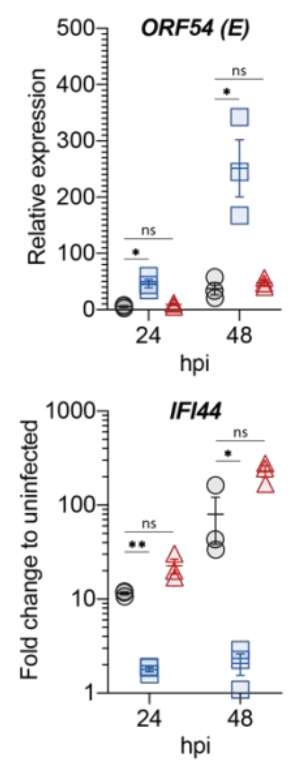

O WT

$\square$ cGAS-KO

$\triangle$ MAVS-KO
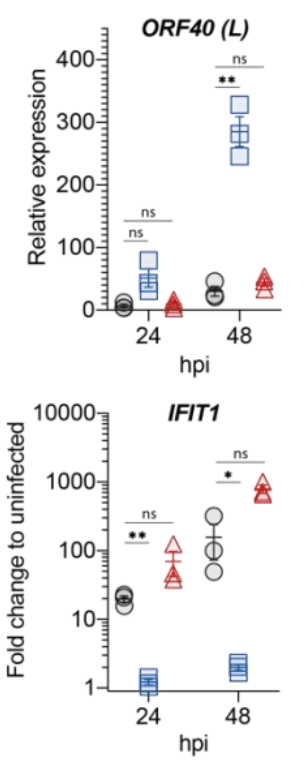

B

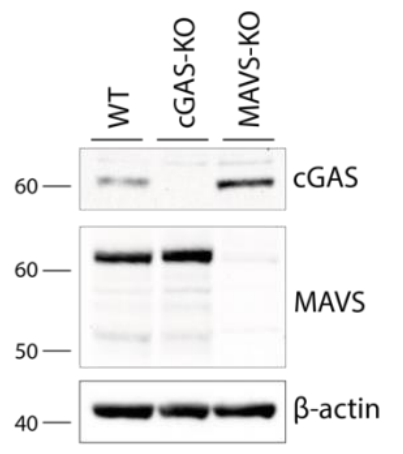

Figure 1: cGAS induces type I IFNs in response to VZV infection.
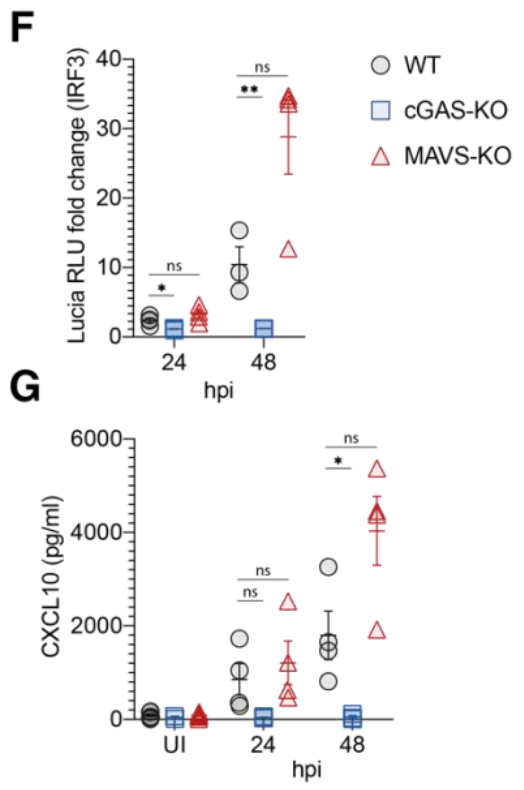

(A) Schematic detailing the experimental procedure for infection of PMA-differentiated THP1 cells with VZV by transwell assay. See text for details. (B) Immunoblot of WT, cGAS KO, and MAVS KO THP1 cells used in (C-G). (C) THP1 cells of the indicated genotypes were mock infected or infected with VZV for 24 and 48 hours using the transwell assay described in (A). Infected cells were quantified by surface staining for VZV-gE/gl and flow cytometry analysis. See Figure S4 for gating. (D) RT-qPCR analysis of VZV ORF63 (immediate early (IE) gene), ORF54 (early (E) gene), and ORF40 (late (L) gene) transcripts in cells infected as in (C). (E) RT-qPCR analysis of IFNB1, IFI44, and IFIT1 expression in cells infected as in (C). (F) Activity of Lucia luciferase (secreted under an IRF3-dependent promotor) was determined in supernatants of cells infected as in (C) by QUANTI-Luc assay. (G) Concentrations of CXCL10 in supernatants of cells infected as in (C) were determined by ELISA.

Panels $(C),(F)$, and $(G)$ show pooled data from four independent biological repeats $(n=4 \pm S E M)$. Panels $(D)$ and (E) show pooled data from three independent biological repeats $(n=3 \pm S E M)$. Statistical analysis in panels (C), (D), $(F)$, and $(G)$ was paired t-tests and in panel $(E)$ paired ratio t-tests. ${ }^{* *}=p<0.01,{ }^{*}=p<0.05$, Ul: uninfected, hpi: hours post infection, WT: wild type, KO: knockout. Panel (B) is representative of two experiments.

See also Figures S1, S2, S3 and S4. 
A
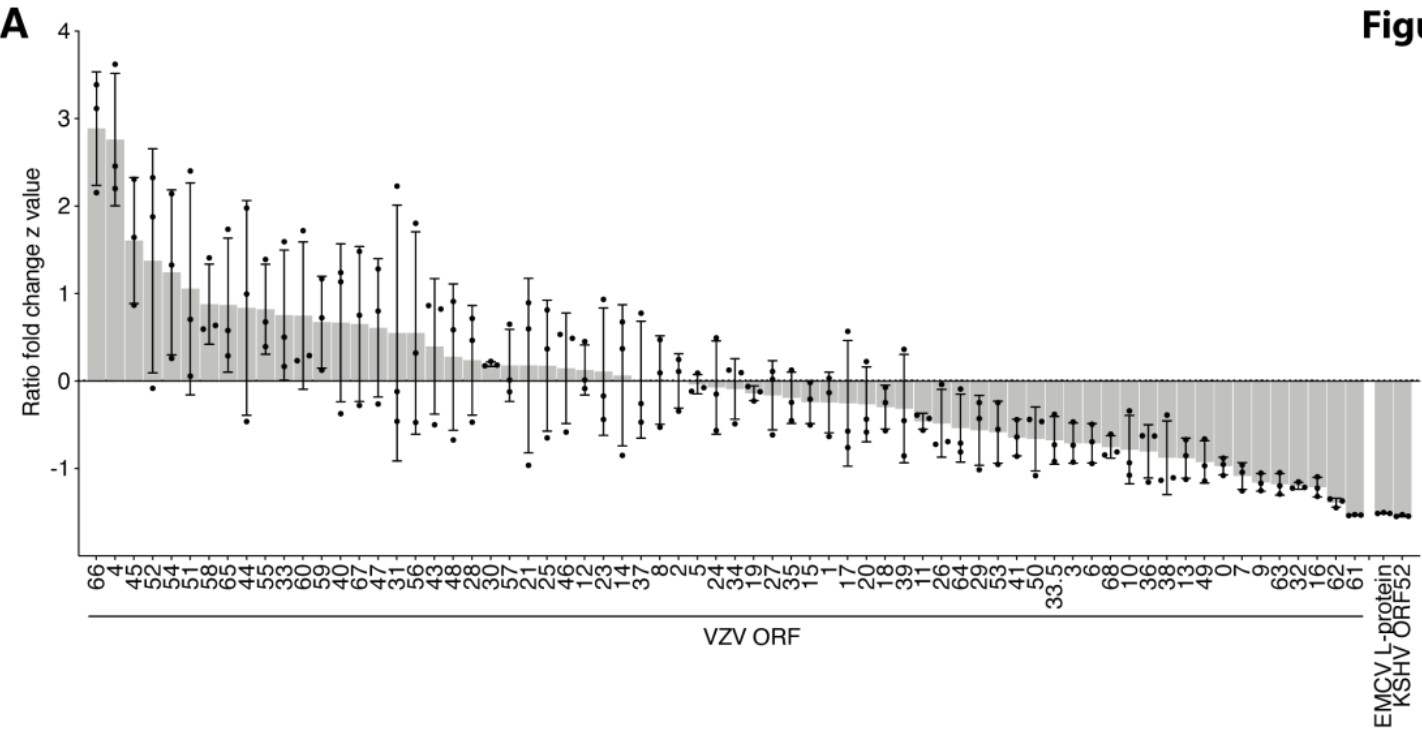

B

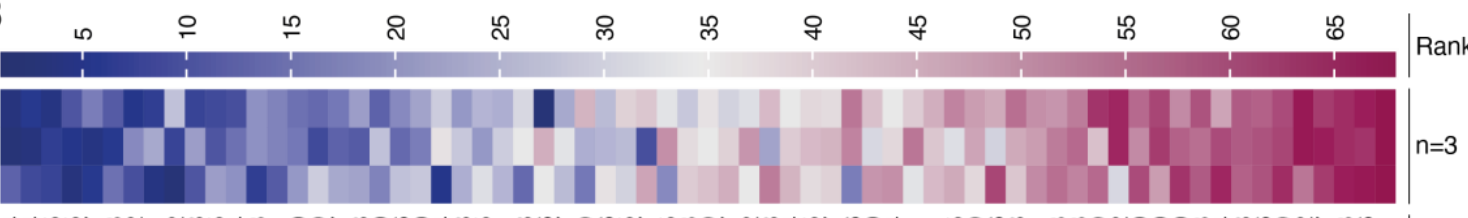

小

C

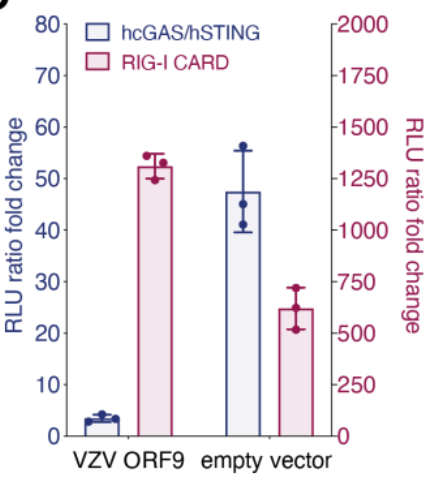

D $\quad$ THP1

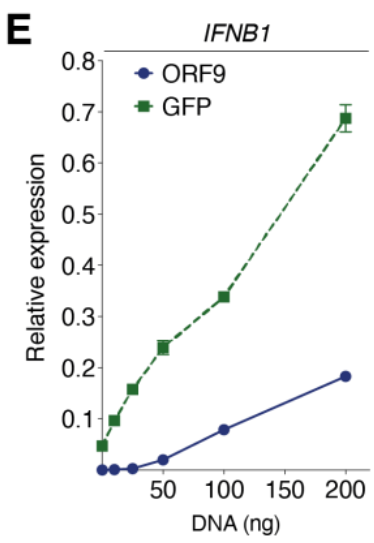

Figure 2

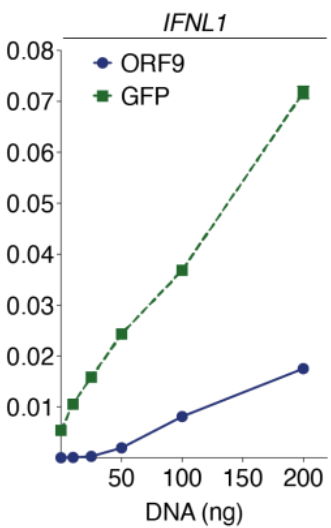

Figure 2. Screening of VZV ORFs identifies ORF9 as an antagonist of cGAS-mediated DNA sensing.

(A) HEK293T cells were transfected with p125-F-Luc, pRL-TK, expression plasmids for human cGAS and human STING, as well as expression plasmids for individual VZV ORFs. The next day luciferase activity was determined. For each ORF a Z-value was calculated and ORFs were sorted in descending order. (B) The experiment shown in

(A) was repeated three times and for each ORF in each experiment a rank was determined (highest Z-value = rank 1). Ranks are displayed as a heatmap. The order of the ORFs in the heatmap was determined by their average rank. (C) HEK293T cells were transfected as in (A) with expression constructs for VZV ORF9 or empty vector. In parallel, reporter expression was stimulated by co-transfection of the RIG-I-CARD plasmid instead of hcGAS and hSTING. A luciferase fold change was calculated to unstimulated cells. (D) Immunoblot analysis of THP1 cells stably transduced with either VZV ORF9-V5 or GFP-V5. (E) The same cells as in (D) were differentiated with PMA and transfected with the indicated doses of dsDNA. Expression of IFNB1 and IFNL1 mRNAs was assessed by RTqPCR. Graphs show expression relative to GAPDH.

Panel $(A)$ is representative of three independent experiments, which are summarised in panel (B). Panels (C-E) are representative of two independent experiments. Data points are technical triplicates with mean and standard deviation $(A, C)$ or the average of technical duplicates with range $(E)$.

See also Figure S5. 
A

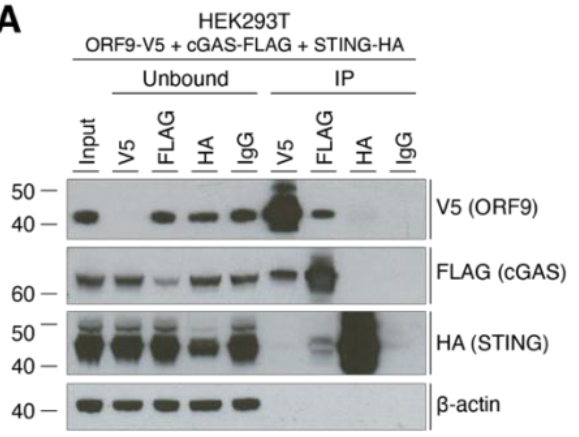

B

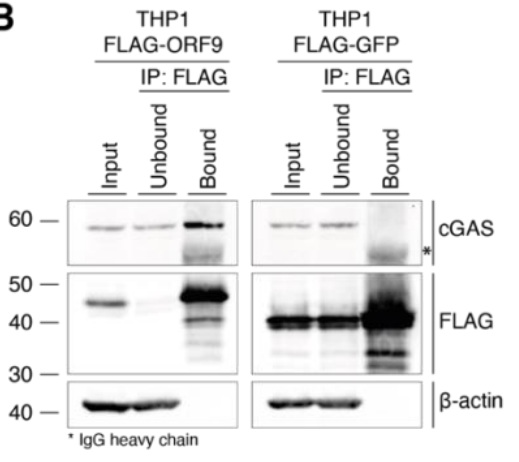

Figure 3

D
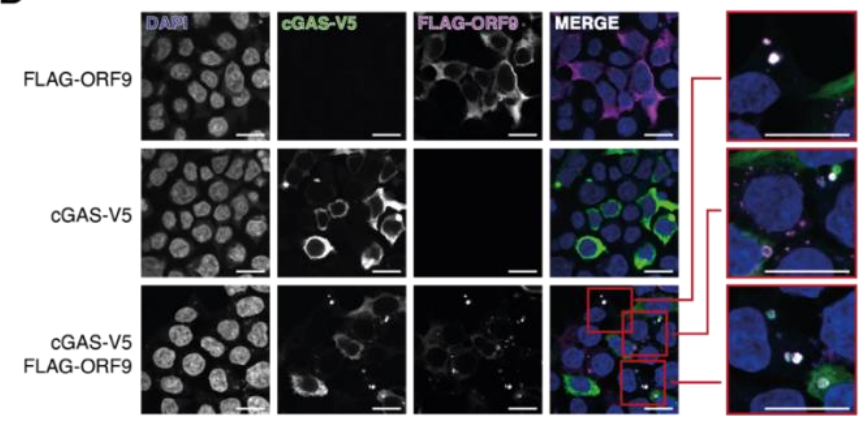

G

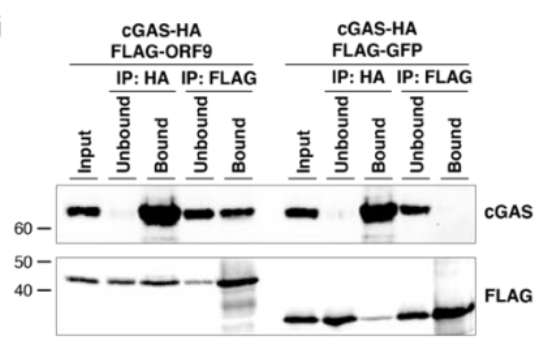

E

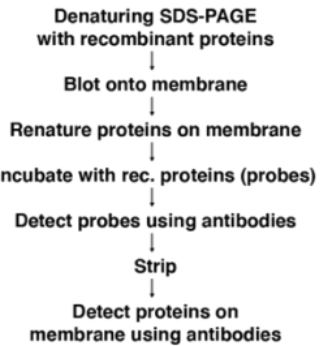

F

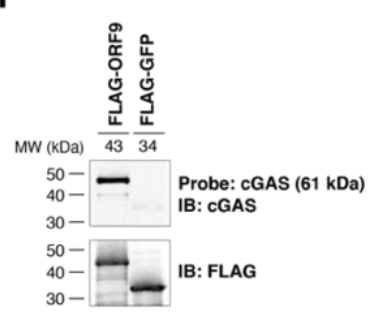

\section{Figure 3. ORF9 interacts with cGAS.}

(A) HEK293T cells were transfected with expression plasmids for ORF9-V5, human cGAS-FLAG, and human STING-HA. The next day, cells were lysed, and overexpressed proteins were immunoprecipitated with $\alpha$-V5, $\alpha$ FLAG, $\alpha-H A$, or IgG isotype control antibody. Input, unbound and IP fractions were subjected to immunoblotting using the indicated antibodies. (B) THP1 monocytes stably transduced with either VZV FLAG-ORF9 or FLAG-GFP were PMA-differentiated overnight. The next day, cells were lysed and ectopically expressed proteins were immunoprecipitated using $\alpha-F L A G$ antibody. Input, unbound and IP fractions were subjected to immunoblotting using the indicated antibodies. (C) HaCaT cells and PMA-differentiated THP1 cells were infected with WT VZV or VZVORF9-V5 through co-culture with infected MeWo cells for 48 hours. Cells were lysed and ORF9 was immunoprecipitated using $\alpha-\mathrm{V} 5$ antibody. Input and IP fractions were subjected to immunoblotting using the indicated antibodies. (D) HEK293T cells were seeded onto glass coverslips and were transfected with human cGAS-V5, FLAG-ORF9, or both together. The next day, cells were fixed, permeabilised and stained using $\alpha$-V5FITC, rabbit- $\alpha$-FLAG, and goat- $\alpha$-rabbit-AF647 antibodies, and DAPI. Mounted coverslips were imaged using confocal microscopy. Scale bars: $15 \mu \mathrm{m}$. (E) Outline of the far western protocol. (F) Far western analysis of cGASORF9 interaction. Recombinant FLAG-ORF9 and FLAG-GFP protein were run on an SDS-PAGE gel and transferred to a membrane. After renaturation of proteins, the membrane was incubated with recombinant human cGAS as a probe, which was detected using $\alpha$-cGAS antibody. After stripping, the proteins on the membrane were detected using $\alpha$-FLAG antibody. (G) Recombinant human cGAS-HA was mixed with recombinant FLAG-ORF9 or FLAG-GFP. Proteins were immunoprecipitated using $\alpha-H A$ and $\alpha-F L A G$ antibodies. Input, unbound and IP fractions were analysed by immunoblotting. Recombinant proteins used in $(F)$ and $(G)$ are shown in Figure S6A.

Panels $(A)$ and $(G)$ are representative of two independent experiments. Panels $(B),(D)$, and $(F)$ are representative of three independent experiments. Panel $(C)$ is representative of two (HaCaT) and three (THP1) independent experiments.

See also Figure S6. 
A

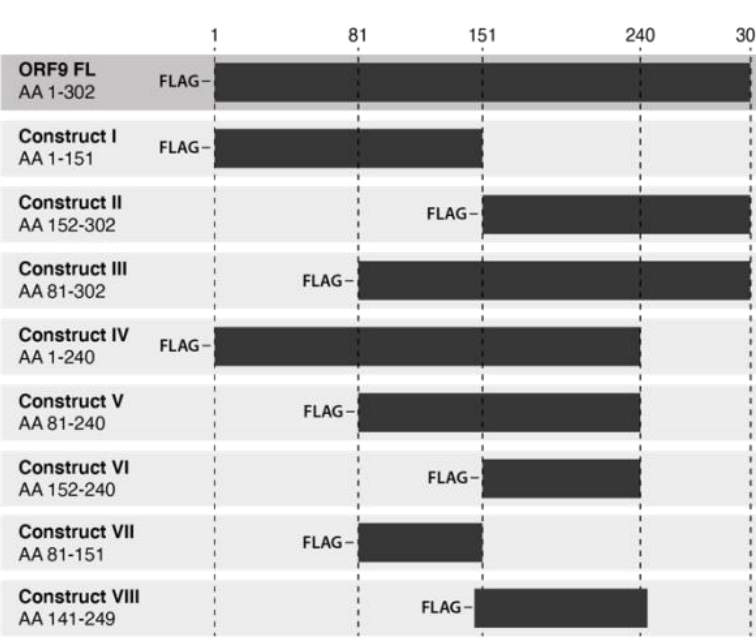

B

Figure 4

C Disordered region
a-helix
$\beta$-sheet
Crystal structure available HSV1 VP22: PDB 4XAL MHV68 ORF52: PDB 2OA5

VZV ORF9

$$
\text { HSV-1 VP22 }
$$

KSHV ORF52

MHV68 ORF52
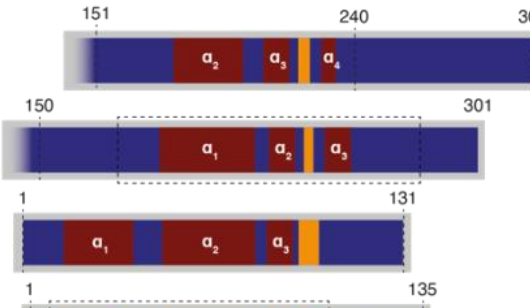

$a_{1} \quad a_{2} \quad a_{3} \quad a_{4}$

302

D
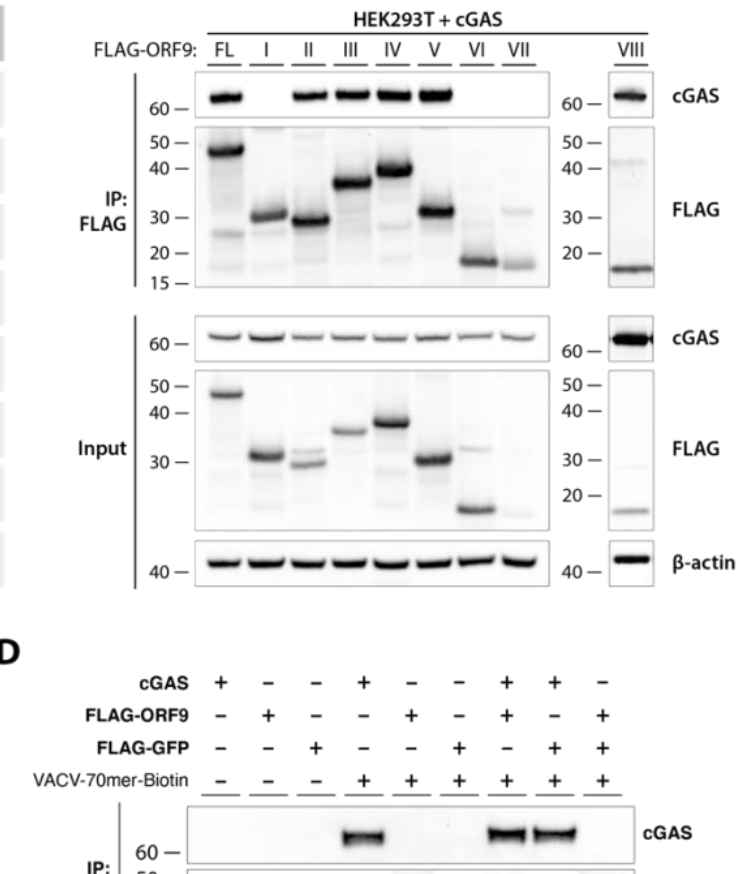

Streptavidin

40

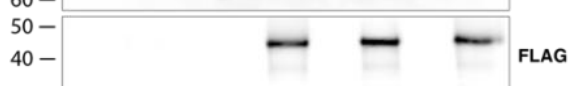

E

$\mathbf{F}$
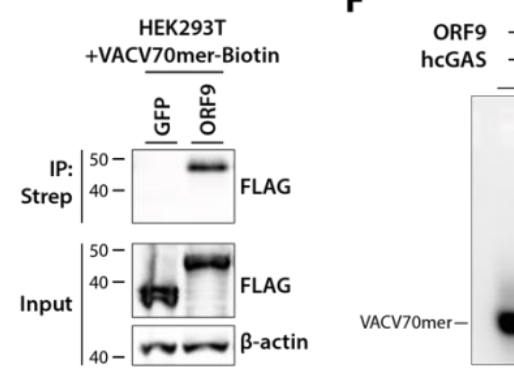

Figure 4: VZV ORF9 binds DNA.

(A) Schematic detailing ORF9 truncation mutants used in (B). (B) HEK293T cells were transfected with expression plasmids for human cGAS and ORF9 truncation mutants. The next day cells were lysed and ORF9 proteins were immunoprecipitated using $\alpha$-FLAG antibody. IP fractions were subjected to immunoblotting using the indicated antibodies. (C) Schematic detailing predicted structural features of VZV ORF9 in relation to predicted and crystal structure-derived features in HSV-1 VP22, KSHV ORF52, and MHV68 ORF52. See text for details. (D) Recombinant cGAS, FLAG-ORF9, FLAG-GFP, and biotinylated VACV70mer dsDNA were incubated in the indicated combinations. Lysates were precipitated using streptavidin beads. Fractions were analysed by immunoblotting. (E) HEK293T cells were transfected with expression plasmids for FLAG-GFP or FLAG-ORF9. The next day cells were lysed, and the lysate was spiked with biotinylated VACV70mer dsDNA. DNA was precipitated using streptavidin beads and fractions were subjected to immunoblotting using the indicated antibodies. (F) VACV70mer dsDNA was incubated with indicated proteins and analysed by agarose gel EMSA. Triangles indicate concentrations of ORF9 $(0.7,1.4,2.9,5.7,8.6 \mu \mathrm{M})$, cGAS $(0.6,1.1,2.2,4.5,6.7 \mu \mathrm{M})$, and ORF9 (as before) in the presence of $4.5 \mu \mathrm{M}$ cGAS. Recombinant proteins used in (D) and (F) are described in Figures S6A and S6B, 
bioRxiv preprint doi: https://doi.org/10.1101/2020.02.11.943415; this version posted April 18, 2021. The copyright holder for this preprint (which was not certified by peer review) is the author/funder, who has granted bioRxiv a license to display the preprint in perpetuity. It is made available under aCC-BY-NC-ND 4.0 International license.

A

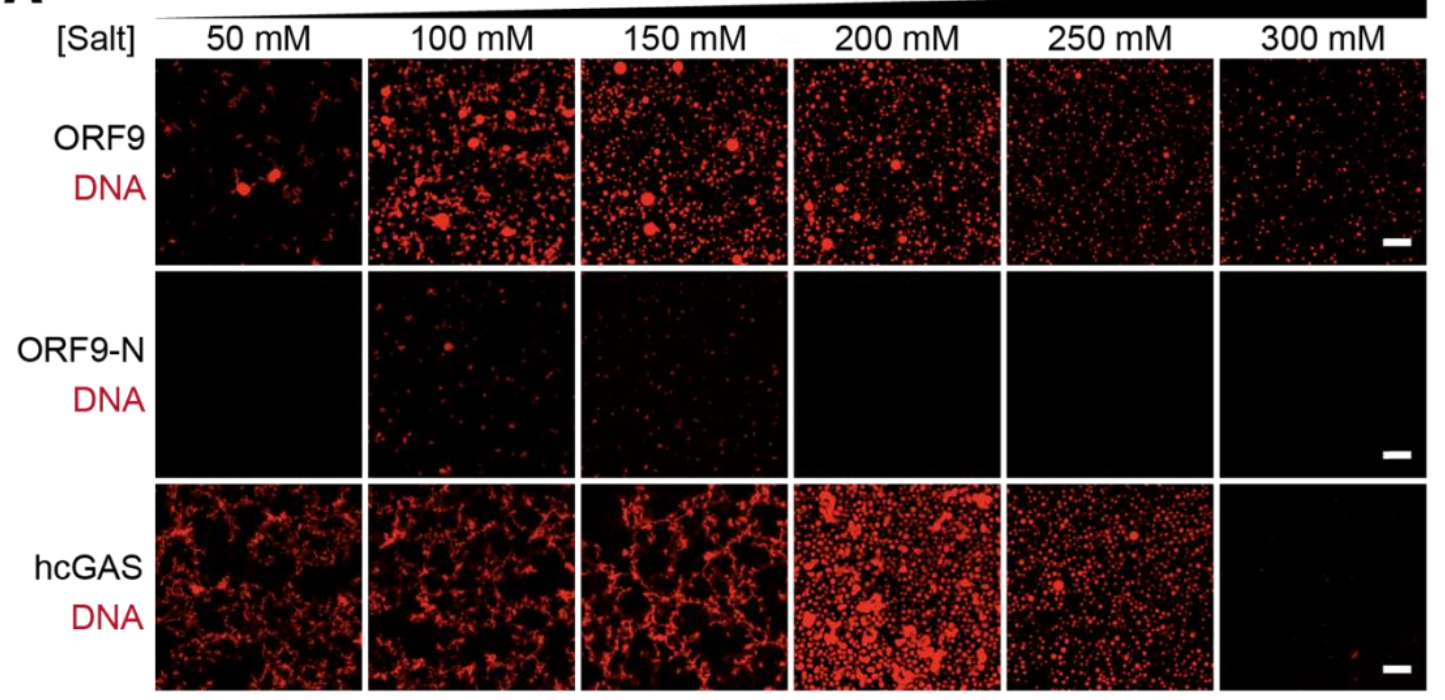

B

Ionic strength

Figure 5

lonic strength 


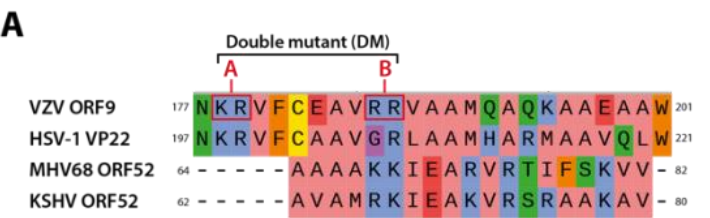

B

D

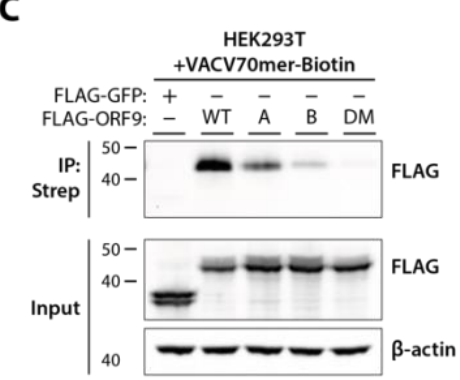

D

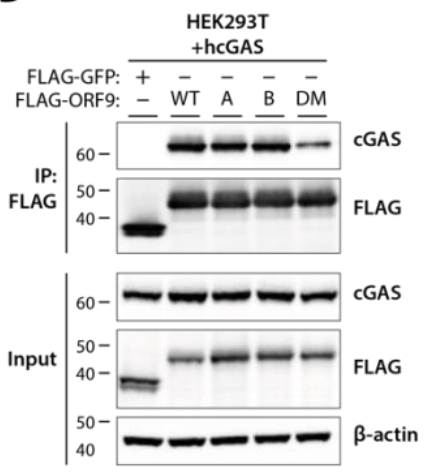

Figure 6

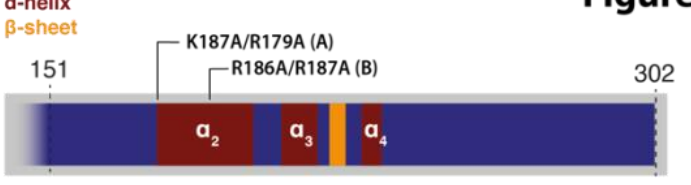

$\mathbf{E}$

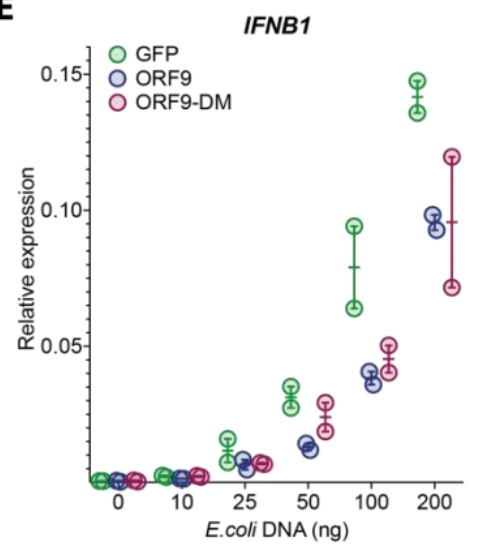

$\mathbf{F}$
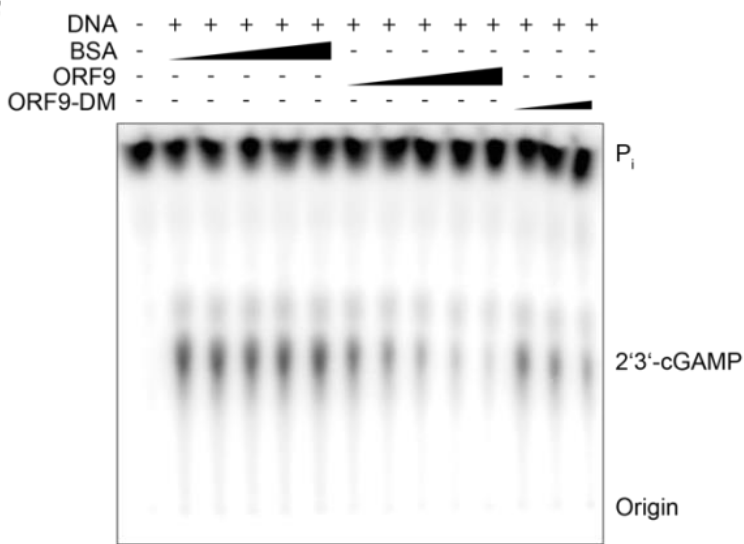

$\mathbf{G}$

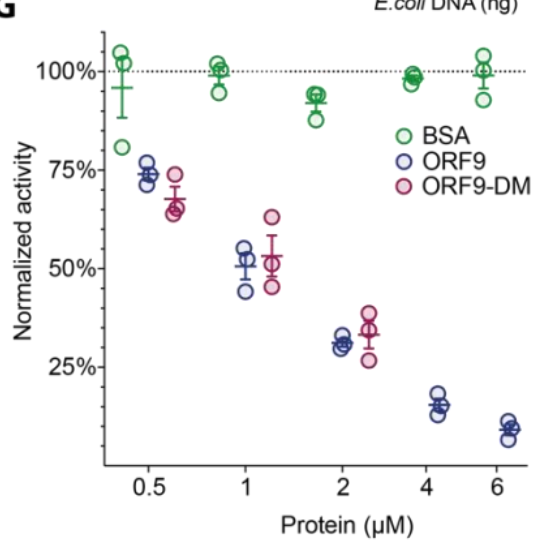

Figure 6: ORF9 inhibits cGAS' catalytic activity.

(A) Protein sequence alignment of VZV ORF9, HSV-1 VP22, KSHV ORF52, and MHV68 ORF52. Residues selected for mutagenesis in ORF9 are highlighted in red. Residue colouring represents physico-chemical properties (green: hydrophilic, blue: positive, coral: aliphatic/hydrophilic, orange: aromatic, yellow: cysteine, red: negative, purple: conformationally special). See text for details. (B) Schematic detailing position and identity of residues mutated in C-G. (C) HEK293T cells were transfected with expression plasmids for FLAG-GFP or FLAG-ORF9 as indicated. The next day cells were lysed, and the lysate was spiked with biotinylated VACV70mer dsDNA. DNA was precipitated using streptavidin beads and proteins were analysed by immunoblotting using the indicated antibodies. (D) HEK293T cells were transfected with expression plasmids for human cGAS and FLAG-GFP or FLAG-ORF9. The next day cells were lysed, and proteins were immunoprecipitated using $\alpha$-FLAG antibody and analysed by immunoblotting using the indicated antibodies. (E) THP1 cells stably transduced with GFP, ORF9, or ORF9-DM were transfected with the indicated amounts of E.coli dsDNA. The next day, IFNB1 expression was assessed by RT-qPCR. (F) In vitro cGAS activity assay. Recombinant hcGAS was incubated with ATP, GTP, and radioactive a32P-ATP at $37^{\circ} \mathrm{C}$ with addition of dsDNA and other proteins as indicated (triangles represent concentrations of $0.5,1,2,4,6 \mu \mathrm{M})$. ORF9-DM was tested only at 0.5, 1, $2 \mu \mathrm{M}$. Reactions were treated with calf-intestinal phosphatase and products were analysed by thin-layer chromatography and phosphorimaging. (G) Signal intensities from (F) were determined by densitometry analysis and normalized to the average BSA signal. Recombinant proteins used in $(F)$ and $(G)$ are described in Figure S6B.

Data shown in panel $(C)$ are representative of two independent experiments. Data shown in panels (D), (E), and $(F)$ are representative of three independent experiments. Panel $(G)$ shows pooled data from three independent experiments ( $\mathrm{n}=2+/$ - range).

See also Figure S6. 


\section{MATERIALS AND METHODS}

649

650

651

652

653

654

655

656

657

658

659

660

661

662

663

664

665

666

667

668

669

670

671

672

673

674

675

676

677

678

679

680

681

682

683

684

685

686

687

688

689

690

691

\section{Cells}

All cells were cultured at $37^{\circ} \mathrm{C}$ and $5 \% \mathrm{CO}_{2}$ and checked regularly for mycoplasma contamination. Adherent cells were passaged using Trypsin-EDTA $(0.25 \%)$ dissociation reagent (Gibco) at appropriate confluence. FCS was obtained from SigmaAldrich. HEK293 and HEK293T cells (gifts from Caetano Reis e Sousa, The Francis Crick Institute, UK) were maintained in DMEM (Sigma-Aldrich) containing $4.5 \mathrm{~g} / \mathrm{L}$ glucose, supplemented with $10 \%$ (v/v) FCS and 2mM L-glutamine (Gibco) (DMEM complete). HEK293-ISRE-Firefly luciferase reporter cells (clone 3C11) were described previously (Bridgeman et al., 2015). HaCaT cells were a gift from Leonie Unterholzner (Lancaster University, UK) and were maintained in DMEM complete medium. MeWo cells were a gift from Graham Ogg (University of Oxford, UK) and were maintained in MEM supplemented with 10\% (v/v) FCS, 2mM L-glutamine (Gibco), 1x Non-essential amino acids (Gibco), and $1 \mathrm{mM}$ sodium pyruvate (Gibco). THP1 Dual cells (WT, MyD88-KO, and IFNAR2-KO) were from Invivogen. STING-KO and TBK1-KO THP1 cells were a gift from Soren Paludan (Aarhus University, Denmark) (Holm et al., 2016). MAVS-KO, cGAS-KO, and IRF3-KO cells were generated as described below. All THP1 cell lines were maintained in RPMI (Sigma Aldrich) supplemented with $10 \%$ (v/v) FCS and 2mM L-glutamine (Gibco).

\section{Viruses}

VZV rOka (cosmid-derived recombinant pOka) was a gift from Jeffrey Cohen (NIH, Bethesda, USA) (Cohen and Seidel, 1993). The virus was maintained in monolayers of MeWo cells. In brief, monolayers of infected cells were monitored microscopically for cytopathic effect. Cells that showed high level of infection were detached and infected cells were mixed at appropriate ratios $(1: 2-1: 4)$ with uninfected cells and re-plated. Aliquots of infected cells were cryopreserved in freezing medium (90\% FCS, $10 \%$ DMSO), stored in liquid nitrogen, and thawed for experiments. VZVORF9-V5 is a recombinant virus, in which a C-terminal V5 tag was added to the coding sequence of ORF9. The virus was a kind gift from Catherine Sadzot (University of Liege, Belgium) (Riva et al., 2013).

\section{Plasmids}

The p125-Luc plasmid ( $h$ IFNB1 promotor firefly luciferase) was a gift from T. Fujita (Kyoto University, Japan) (Yoneyama et al., 2004). pRL-TK was from Promega. RIG-ICARD was a gift from Andreas Pichlmair (Technical University Munich, Germany). pCMV-VSV-G was a gift from Bob Weinberg (Addgene number 8454). The lentiviral packaging plasmid p8.91 was a gift from Greg Towers (University College London). pGag-eGFP was from the NIH AIDS reagent programme (number 11468). pX458/Ruby/MAVS was described previously (Hertzog et al., 2018). pCMV-3tag-KSHV- 
692 ORF52 was a kind gift from Fanxiu Zhu (Florida State University, USA). $693 \mathrm{pX} 458 /$ Ruby/IRF3 was created as described for the MAVS-targeting plasmid. A sgRNA 694 targeting exon 3 of the IRF3 locus was designed using the MIT algorithm 695 (crispr.mit.edu) and cloned into the px458 vector. The pLenti6.3/EMCV-L-V5 plasmid 696 was described previously (Hertzog et al., 2018).

697

698

699

700

701

702

703

704

705

706

707

708

709

710

711

712

713

714

715

716

717

718

719

720

721

722

723

724

725

726

727

728

729

730

731

732

733

734

\section{Cloning}

Mammalian expression plasmids for hcGAS and hSTING were created by PCRamplification using THP1 cDNA and ligation into the gateway entry vector pCR8. Coding sequences were shuttled into expression vectors (pLenti6.3/C-V5, pcDNA3.2/C-FLAG, pcDNA3.2/C-HA) via Gateway recombination. Mammalian expression plasmids for eGFP (pLenti6.3/puro/N-FLAG, pDEST31/N-FLAG) were created in a similar way by PCR amplification from pGag-eGFP introducing a stop codon. VZV ORF9 expression plasmids (pLenti6.3/puro/N-FLAG, pDEST31/N-FLAG) were created by amplification of ORF9 with a stop codon and gateway recombination. Expression plasmids for ORF9 truncation mutants (pLenti6.3/puro/N-FLAG) were created by PCR amplification of the respective coding sequence with a start and stop codon. ORF9 mutant plasmids were generated using site directed mutagenesis (QuikChange II Site-Directed Mutagenesis Kit, Agilent). GST-fusion bacterial expression vectors for FLAG-ORF9, FLAG-GFP, and hcGAS-HA were created by PCR-amplification of the coding sequences from existing plasmids and restriction enzyme cloning into pGEX6P1. pGEX6P1 and pGEX6P1-hcGAS were a kind gift from Martin Reijns (University of Edinburgh, UK). Further bacterial expression vectors for FLAG-ORF9 and FLAG-ORF9-DM were created by restriction enzyme cloning of coding sequences into the pET28a bacterial expression vector (kind gift from Simon Davis, University of Oxford). Bacterial expression vectors for ORF9 and ORF9-N were created by restriction enzyme cloning of coding sequences into a custom 6xHis-SUMO bacterial expression vector (Zhou et al., 2018). All primer sequences are listed in Table S1.

\section{Immunostimulatory dsDNA}

E. coli dsDNA was from Invivogen. A 70bp immunostimulatory dsDNA fragment from VACV was described previously (Unterholzner et al., 2010). Two complementary oligos were synthesised (Sigma Aldrich) and combined at equal molar ratio. The solution was heated to $95^{\circ} \mathrm{C}$ and allowed to anneal by cooling to RT.

Forward sequence:

5'-ccatcagaaagaggtttaatattttgtgagaccatcgaagagagaaagagataaaactttttacgact-3'-TEG-

Biotin

Reverse sequence:

5'-agtcgtaaaaaagttttatctctttctctcttcgatggtctcacaaaaa-tattaaacctcttctgatgg-3' 
The 100bp dsDNA used for the phase separation assay was described previously (Zhou et al., 2021). DNA oligos were synthesised (Integrated DNA Technologies) and double-stranded DNA was prepared by annealing two complementary oligos.

738 Forward sequence:

739 5'-

740 ACATCTAGTACATGTCTAGTCAGTATCTAGTGATTATCTAGACATACATCTAGTA

741 CATGTCTAGTCAGTATCTAGTGATTATCTAGACATGGACTCATCC-3'

742 Reverse sequence:

743 5'-

744 GGATGAGTCCATGTCTAGATAATCACTAGATACTGACTAGACATGTACTAGATGT

745 ATGTCTAGATAATCACTAGATACTGACTAGACATGTACTAGATGT-3'

\section{Antibodies}

For immunoblot: beta-actin-HRP (AC-15, Sigma Aldrich), FLAG-HRP (clone M2, Sigma Aldrich), V5-HRP (R961-25, Invitrogen), RIG-I (Alme1, Calteg Medsystems), VZV gE/GI (MAB8612, GE Healthcare), VZV ORF62 (C05107MA, Meridian Life Science), pSTAT1 (Y701) (58D6, CellSignaling Technology), STAT1 (42H3, CellSignaling Technology), pSTAT2 (D3P2P, CellSignaling Technology), STAT2 (D9J7L, CellSignaling Technology), hcGAS (D1D3G, CellSignaling Technology), hSTING (D2P2F, CellSignaling Technology), MyD88 (D80F5, CellSignaling Technology), TBK1 (D1B4, CellSignaling Technology), MAVS (ALX-210-929-C100, ENZO Life Science), IRF3 (D6I4C, CellSignaling Technology), VZV ORF9 (polyclonal rabbit serum, kind gift from Catherine Sadzot (University of Liege, Belgium) (Riva et al., 2013), donkey-anti-mouse-HRP (NA931, GE Healthcare), donkey-anti-rabbit-HRP (NA931, GE Healthcare). For IP: FLAG (clone M2, Sigma Aldrich, V5 (680602, Biolegend), HA (2-2.2.14, Invitrogen). For IF: V5-FITC (R963-25, Invitrogen), FLAG (D6W5B, CellSignaling Technology), goat-anti-rabbit-AF647 (A21246, Invitrogen). For FACS: VZV-gE/gI (see above) was conjugated to FITC using FITC Conjugation Kit (Abcam).

\section{VZV ORF Library}

Primers for PCR amplification of individual VZV ORF sequences were designed based on an annotated VZV genome sequence (GenBank accession: AB097933.1). Forward primers included a Kozak sequence (GCCGCC), added before the start codon of an ORF. Reverse primers excluded the Stop codon to allow for addition of a C-terminal tag through the vector (see below). Primer sequences are listed in Table S2. The PCR template was generated by extracting RNA from VZV infected MeWo cells using QIAshredder (Qiagen) and RNeasy Mini Kit (Qiagen). The RNA was reverse transcribed into cDNA using SuperScript II Reverse Transcriptase (Invitrogen). For some ORFs, DNA from infected MeWo cells extracted with DNeasy Blood and Tissue Kit (Qiagen) served as the PCR template. PCR products were generated using Phusion High-Fidelity DNA Polymerase (New England Biolabs) or Herculase II Fusion 
DNA Polymerase (Agilent Technologies). PCR reactions were analysed by agarose gel electrophoresis and PCR products of the predicted size were extracted from the gel using QIAquick Gel Extraction Kit (Qiagen). Fragments were ligated into the

782

783

784

785

786

787

788

789

790

791

792

793

794

795

796

797

798

799

800

801

802

803

804

805

806

807

808

809

810

811

812

813

814

815

816

817

818

819

820

821

822

823 pCR8/TOPO Gateway entry vector (Invitrogen). Plasmid DNA from single clones of transformed E.coli (New England Biolabs) was analysed for correct orientation of the insert by Sanger sequencing. Inserts were then shuttled into pLenti6.3/TO/V5-DEST (Invitrogen) using LR Clonase II Enzyme Mix (Invitrogen) for Gateway recombination. All clones with the pLenti6.3/TO/V5 backbone were propagated in recombinationdeficient Stbl3 bacteria (Invitrogen).

Cloning of the entire coding sequence of ORF22 (8256 bp) was unsuccessful using various amplification and cloning technologies. Therefore, two Gateway entry vectors encoding individual segments within the ORF22 coding sequence (ORF22A: nt 22194029, ORF22B: nt 4012-6114, described previously (Uetz et al., 2006)) were used (kind gift from Jurgen Haas, University of Edinburgh, UK). Expression vectors with the pLenti6.3/TO/V5 backbone were generated as described above.

To validate expression, HEK293T were seeded at $5 \times 10^{5}$ cells per well in 12-well plates. The next day, cells were transfected with 600ng VZV ORF or control expression plasmids using $3 \mu$ l Lipofectamine 3000 (Invitrogen) per well. 24 hours later, cells were lysed in RIPA buffer (10mM TRIS-HCl pH8, $140 \mathrm{mM} \mathrm{NaCl}, 1 \%$ Triton-X 100, 0.1\% SDS, $0.1 \%$ sodium deoxycholate, $1 \mathrm{mM}$ EDTA, $0.5 \mathrm{mM}$ EGTA). Lysates were clarified by centrifugation and $30 \mu \mathrm{g}$ protein was subjected to immunoblotting.

\section{Luciferase reporter assays}

HEK293T cells were seeded at $3.5 \times 10^{4}$ cells per well in 96-well plates. On the following day, cells were transfected with the following plasmids using Lipofectamine 2000 (Invitrogen): 20ng p125-F-Luc, 5ng pRL-TK, 1ng hcGAS, 25ng hSTING, and 50ng of a VZV ORF. Alternatively, cells were transfected with 5 ng RIG-I-CARD plasmid instead of CGAS and STING plasmids. The next day, expression of firefly and renilla luciferases was assessed using Dual Luciferase assay system (Promega).

Activity of the secreted Lucia luciferase under IRF3 promoter control (THP1 Dual cells) was assessed using QuantiLuc substrate (Invivogen) according to manufacturer's instructions.

\section{Lentivirus Production and Transduction}

$1.2 \times 10^{7}$ HEK293T cells were seeded in $15 \mathrm{~cm}$ cell culture dishes. The next day, cells were transfected with $9 \mu \mathrm{g}$ lentiviral expression plasmids harbouring the gene of

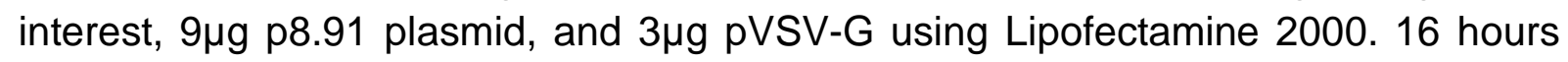
later, medium was replaced with fresh growth medium. 24 hours later, lentivirus containing supernatant was harvested, clarified by centrifugation, and stored at $4^{\circ} \mathrm{C}$. Cells were overlaid with fresh growth medium. 8 hours later, supernatants were harvested again and pooled with previous supernatants. After 16 hours, lentivirus containing supernatants were harvested for a third time. Pooled supernatants from all three harvests were filtered through a $0.45 \mu \mathrm{m}$ filter, aliquoted into cryovials and stored 
at $-80^{\circ} \mathrm{C}$. For transduction of cells, polybrene (Sigma Aldrich) was added to lentiviral supernatants to a final concentration of $8 \mu \mathrm{g} / \mathrm{ml}$. THP1 cells were pelleted and resuspended in lentiviral supernatant containing polybrene. The next day, cells were pelleted and resuspended in fresh growth medium. After overnight incubation, cells were once again pelleted and resuspended in growth medium containing $10 \mu \mathrm{g} / \mathrm{ml}$ blasticidin or $1 \mu \mathrm{g} / \mathrm{ml}$ puromycin (both Gibco) depending on which vector was used. Surviving cells were used for experiments.

\section{THP1 Knockout Cell Generation}

For generation of knockout cells using CRISPR/Cas9 technology the pX458-mRuby plasmid encoding the Cas9 protein, the sgRNA, and mRuby was used (Hertzog et al., 2018). $1 \times 10^{7}$ THP1 cells were transiently transfected with $50 \mu \mathrm{g}$ of respective $\mathrm{pX} 458$ plasmids using Lipofectamine LTX (Invitrogen) and incubated overnight. The next day cells were stained with $10 \mu \mathrm{g} / \mathrm{ml}$ DAPI in PBS for $10 \mathrm{~min}$. After resuspension of cells in growth medium, live, mRuby-positive cells were FACS-sorted into eppendorf tubes containing growth medium with $20 \%$ FCS. The suspension of sorted cells was diluted with special growth medium (50\% conditioned THP1 medium, 40\% fresh RPMI with $10 \%$ FCS, $10 \%$ additional FCS) to one or three cells per $200 \mu \mathrm{l} .200 \mu$ l cell suspension were then dispensed into each well of a 96-well plate (one or three cells per well). The cells were incubated for several weeks until clones grew out. analysis. For functional validation of MAVS and IRF3 knockout cells, $1.5 \times 10^{5}$ cells were seeded per well into 96-well plates in growth medium containing 10ng/ml PMA (SigmaAldrich). The next day cells were transfected with 5ng IVT-RNA ( or 15ng E.coli DNA (Invivogen) using Lipofectamine 2000 (Invitrogen). 24 hours later, IFN in supernatants was measured using the type I IFN bioassay. For functional validation of cGAS knockout cells, cells were stimulated in the same way and activity of Lucia luciferase (under IRF3 promoter control) was assessed in supernatants. Knockout clones were further analysed for insertions and deletions in their genomic loci. MAVS and IRF3 target regions were PCR-amplified using Herculase II Fusion DNA Polymerase. PCR amplicons were gel extracted (QIAquick Gel Extraction Kit) and sequenced by Sanger sequencing. Sequencing traces were analysed using the TIDE software (https://tide.nki.nl) (Brinkman et al., 2014).

\section{VZV Flow Cytometry Infection Assay}

Cells were washed in FACS tubes with PBS and incubated with LIVE/DEAD Fixable

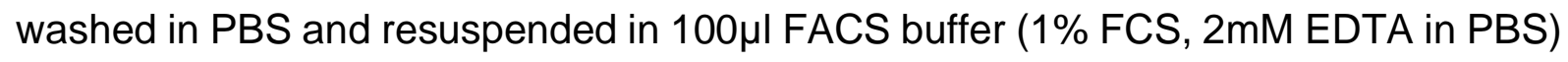
containing 1:500 FITC-coupled antibody against VZV gE/gl complex. Cells were incubated $30 \mathrm{~min}$ at $4^{\circ} \mathrm{C}$. After washing with PBS cells were resuspended in PBS and an equal volume of $8 \%$ methanol-free formaldehyde in PBS was added. Cells were fixed for $15 \mathrm{~min}$ at room-temperature. Cells were washed with PBS, resuspended in 
FACS buffer, and analysed on a Attune NxT Acoustic Focusing Cytometer (Thermo Fisher).

\section{THP1 VZV co-culture Infections}

6.125 $\times 10^{6}$ THP1 cells were seeded per well in 6-well plates in growth medium

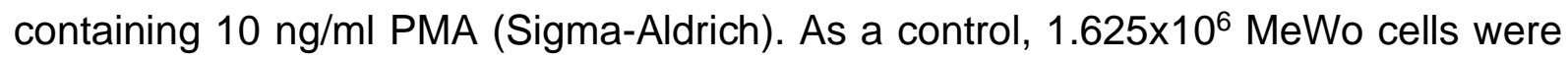
seeded in MEM. Aliquots of uninfected and VZV-infected MeWo cells were thawed, washed, and resuspended in MEM. Cells were counted and their concentration was adjusted to $6.25 \times 10^{5}$ live cells per $\mathrm{ml}$ (for THP1 cells) or $1.25 \times 10^{5}$ live cells per $\mathrm{ml}$ (for MeWo cells) with MEM. Medium was removed from labelled cells and cells were overlaid with $2 \mathrm{ml}$ MeWo $-/+\mathrm{VZV}$ cell suspension. Cells were incubated at $37^{\circ} \mathrm{C}$ for 1 hour. The cell suspension was removed, adherent cells were washed with PBS, overlaid with their respective growth medium and incubated for 48 hours. Supernatants were removed from cells, clarified by centrifugation and stored at $-80^{\circ} \mathrm{C}$. CXCL10 levels were determined using Human CXCL10/IP-10 Quantikine ELISA Kit (R\&D Systems) according to manufacturer's instructions. Cells were washed with PBS and incubated with Trypsin-EDTA until they started to detach. Growth medium was added, cells were resuspended by gentle pipetting and transferred to tubes. Half of the cell suspension were transferred to eppendorf tubes on ice for extraction of RNA and generation of protein lysates, respectively. The cells in those eppendorf tubes were pelleted, washed with PBS, and then lysed either in RLT buffer and processed for RTqPCR or lysed in RIPA buffer and processed for immunoblot analysis.

\section{THP1 transwell VZV infections}

895

896

897

898

899

900

901

902

903

904

905

906

907

908

909

910

PET membrane $1 \mu \mathrm{m}$ transwell inserts (Sarstedt 83.3930.101) were placed with the bottom membrane facing upwards into $15 \mathrm{~cm}$ dishes. Aliquots of uninfected and VZVinfected MeWo cells were thawed, washed, and resuspended in MEM. Cells were counted and their concentration was adjusted to $3 \times 10^{6}$ live cells per ml with MEM. 500 $\mu \mathrm{l}$ cell suspension $\left(1.5 \times 10^{6}\right.$ cells) was pipetted onto the transwell membrane and cells were let adhere in the incubator overnight. 6-well plates were filled with RPMI media and prepared transwells were placed cell-side downwards into the plates, so that the MeWo cells were submerged. $2 \times 10^{6}$ THP1 cells were seeded onto the upward facing side of the transwell membrane in RPMI medium containing $10 \mathrm{ng} / \mathrm{ml}$ PMA. Cells were harvested by trypsinisation after $24 \mathrm{~h}$ and $48 \mathrm{~h}$ and used for downstream analysis. A publication describing this method in more detail is in preparation.

\section{Pulldowns}

$1.4 \times 10^{7}$ HEK293T cells were seeded in $15 \mathrm{~cm}$ cell culture dishes. The next day, cells were transfected with $12.7 \mu \mathrm{g}$ of ORF9-V5, cGAS-FLAG, and STING-HA expression plasmids using Lipofectamine 2000. 16 hours later, cells were lysed in IP buffer $(20 \mathrm{mM}$ TRIS-HCI pH7.4, 100mM NaCl, 1mM EDTA, 0.5\% NP-40, Protease Inhibitor Cocktail (CellSignaling Technology)). After clarification, an aliquot was removed as input 
sample and the lysate was split into four equal volumes. Each aliquot was incubated with $50 \mu$ l Dynabeads Protein G that were coated with $5 \mu \mathrm{g} \alpha$-V5, $\alpha$-FLAG, $\alpha-H A$, or control $\lg G$ antibody for 1 hour under rotation at $4^{\circ} \mathrm{C}$. The supernatant was removed, an aliquot was stored as unbound fraction from each sample, and beads were washed three times in lysis buffer. Input, unbound, and bound fractions were subjected to immunoblotting. For pulldowns from stably transduced THP1 cells, cells were seeded at $2 \times 10^{7}$ per dish in $15 \mathrm{~cm}$ cell-culture dishes in growth medium containing $10 \mathrm{ng} / \mathrm{ml}$ PMA. Lysates were generated the next day and processed as described above. For pulldowns of recombinant proteins, $3 \mu \mathrm{l}$ recombinant cGAS-HA protein was mixed with $1.5 \mu \mathrm{l}$ FLAG-ORF9 or FLAG-GFP in IP buffer. IP was performed as described above.

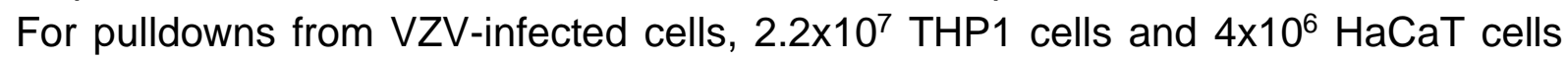
were seeded in $10 \mathrm{~cm}$ dishes (in the presence of $10 \mathrm{ng} / \mathrm{ml}$ PMA for THP1 cells). The next day cells were overlaid with $1.25 \times 10^{7}$ MeWo cells infected with VZV or VZVORF9V5 for 1 hour and afterwards washed with PBS. Lysates were generated 48 hours later as described above.

\section{Far western}

The experimental procedure for far western was based on a protocol previously

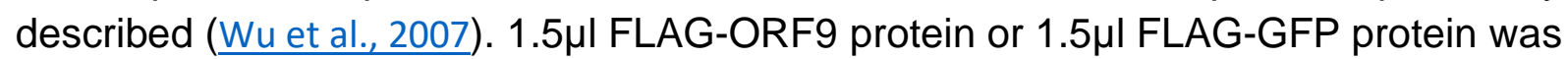
subjected to SDS-PAGE and blotting as described for regular immunoblotting (see below). After transfer, the membrane was incubated for 30min at RT temperature in AC buffer (6M Guanidine $\mathrm{HCl}, 100 \mathrm{mM} \mathrm{NaCl}, 20 \mathrm{mM}$ TRIS-HCl pH 7.6, 0.5mM EDTA, $10 \%$ glycerol, $0.1 \%$ Tween-20, $2 \%$ skim milk powder, $1 \mathrm{mM}$ DTT). The membrane was then incubated in $\mathrm{AC}$ buffers with decreasing concentrations of Guanidine $\mathrm{HCl}(3 \mathrm{M}$, $1 \mathrm{M}, 0.1 \mathrm{M})$ for $30 \mathrm{~min}$ each. For the last incubation the membrane was transferred to $4^{\circ} \mathrm{C}$ and thereafter incubated in $\mathrm{AC}$ buffer free of Guanidine $\mathrm{HCl}$ overnight. The membrane was blocked for 1 hour in 5\% milk powder in PBST (PBS with $0.05 \%$ Tween-20) and then incubated with $10 \mu \mathrm{l}$ recombinant cGAS per $5 \mathrm{ml}$ buffer as probe in 3\% milk powder in PBST for 1 hour. Membranes were washed for 10min with PBST thrice and then incubated with $\alpha$-cGAS antibody for 1 hour. After washing, membranes were incubated with appropriate secondary antibodies, washed again, and imaged. Hereafter, membranes were stripped and re-probed with $\alpha$-FLAG antibody as described for conventional immunoblot.

\section{Immunofluorescence}

1.75 $\times 10^{5}$ HEK293T cells were seeded onto-glass coverslips. The next day, cells were transfected with $250 \mathrm{ng}$ of cGAS-V5 or FLAG-ORF9 expression plasmid using $1.5 \mu \mathrm{l}$ Lipofectamine 3000 per well. Additional coverslips were co-transfected with both plasmids. 24 hours later cells were washed with PBS and fixed with $4 \%$ formaldehyde in cytoskeleton stabilisation buffer (CSB; $10 \mathrm{mM} \mathrm{KCl}, 274 \mathrm{mM} \mathrm{NaCl}, 8 \mathrm{mM} \mathrm{NaHCO}$, $0.8 \mathrm{mM} \mathrm{KH}_{2} \mathrm{PO}_{4}, 2.2 \mathrm{mM} \mathrm{Na}_{2} \mathrm{HPO}_{4}, 4 \mathrm{mM} \mathrm{MgCl}_{2}$, 10mM PIPES, 4mM EGTA, $11 \mathrm{mM}$ glucose) for $15 \mathrm{~min}$. Cells were washed and permeabilised with $0.1 \%$ Triton $X-100$ in 
CSB for $20 \mathrm{~min}$ and incubated with $100 \mathrm{mM}$ glycine in CSB for $10 \mathrm{~min}$ afterwards. Cells were washed with PBS four times and blocked using 1\% BSA and 5\% normal goat

959

960

961

962

963

964

965

966

967

968

969

970

971

972

973

974

975

976

977

978

979

980

981

982

983

984

985

986

987

988

989

990

991

992

993

994

995

996

997

998

999

1000

1001 serum (Invitrogen) in PBS for 1 hour. Coverslips were incubated with primary antibodies in blocking solution for 3 hours at room temperature. Cells were washed three times with PBS and incubated with secondary antibodies in blocking solution for 1 hour. Coverslips were washed with PBS three times and mounted onto glass slides using ProLong Diamond Antifade Mountant with DAPI (Invitrogen). Images were acquired using a Zeiss LSM780 confocal microscopy system (Zeiss).

\section{Recombinant Protein Expression}

\section{Single-step purification of GST-fusion proteins (see Figure S6A)}

BL21-pLysS-Rosetta E.coli were transformed with bacterial expression vectors. A single colony or a glycerol stock from a single colony were used to inoculate a starter culture of $25 \mathrm{ml}$ LB-medium containing $34 \mu \mathrm{g} / \mathrm{ml}$ Chloramphenicol and $100 \mu \mathrm{g} / \mathrm{ml}$ Carbenicillin. A large 400ml LB culture was inoculated using the starter culture. OD600 was measured in intervals and, for VZV ORF9 and GFP, recombinant protein expression was induced by adding $0.1 \mathrm{mM}$ IPTG once OD reached 0.6. Bacteria were grown 3 hours at $37^{\circ} \mathrm{C}$. For expression of recombinant cGAS and cGAS-HA, bacteria were grown to OD 0.8 and chilled to $18^{\circ} \mathrm{C}$. Protein expression was induced by adding $0.4 \mathrm{mM}$ IPTG and bacteria were incubated for 16 hours at $18^{\circ} \mathrm{C}$. For all proteins, bacteria were pelleted at $6,000 \mathrm{~g}$ for $7 \mathrm{~min}$ and the pellet was resuspended in $12 \mathrm{ml}$ lysis buffer (20mM TRIS pH7.4, 500mM NaCl, 0.5mM EDTA, 0.5mM EGTA, 0.5\% NP40, 1:100 Protease Inhibitor Cocktail (CellSignaling Technology)). The suspension was sonicated on ice three times at $20 \%$ amplitude with $15 \mathrm{sec}$ ON and $30 \mathrm{sec}$ OFF (Branson Sonifier). Insoluble material was removed by centrifugation for $15 \mathrm{~min}$ at 24,000g. In the meantime, $150 \mu \mathrm{l}$ Glutathione Sepharose beads (GE Healthcare) were prepared according to manufacturer's instructions. The beads were added to the clarified lysate and incubated under rotation for 4 hours at $4^{\circ} \mathrm{C}$. The beads were pelleted by centrifugation at $500 \mathrm{~g}$ and washed five times with lysis buffer. The beads were washed once in PreScission Cleavage buffer (50mM TRIS pH 7.5, 150mM, $1 \mathrm{mM}$ freshly added DTT) and resuspended in 500 $\mu$ PreScission Cleavage buffer. After addition of $12 \mu \mathrm{l}$ PreScission Protease (GE Healthcare), the suspension was incubated under rotation for 3 hours at $4^{\circ} \mathrm{C}$. The supernatant containing the recombinant protein was separated from beads and stored at $-80^{\circ} \mathrm{C}$. Aliquots from the various purification steps were analysed by SDS-PAGE and subsequent staining of the gel with EZBlue Gel Staining Reagent (Sigma Aldrich). Based on band intensities the protein concentrations were estimated to be ca. $1 \mu \mathrm{g} / \mu \mathrm{l}$ for FLAG-ORF9 and FLAG-GFP, and ca. $0.5 \mu \mathrm{g} / \mu \mathrm{l}$ for cGAS and cGAS-HA.

\section{ORF9 purifications for EMSA and cGAS activity assay (Figure S6B)}

BL21-pLysS-Rosetta E.coli (Novagene) were transformed with bacterial expression vectors. Three fresh, single colonies were used to inoculate a starter culture of $150 \mathrm{ml}$ LB-medium containing $34 \mu \mathrm{g} / \mathrm{ml}$ chloramphenicol and $50 \mu \mathrm{g} / \mathrm{ml}$ kanamycin and grown overnight at $16^{\circ} \mathrm{C}$. Two large $1 \mathrm{~L}$ LB cultures were then inoculated using the starter culture. Bacteria were grown to OD 0.8 and chilled to $16^{\circ} \mathrm{C}$. Protein expression was 
1002

1003

1004

1005

1006

1007

1008

1009

1010

1011

1012

1013

1014

1015

1016

1017

1018

1019

1020

1021

1022

1023

1024

1025

1026

1027

1028

1029

1030

1031

1032

1033

1034

1035

1036

1037

1038

1039

1040

1041

1042

1043

1044

1045

1046

induced by adding $0.5 \mathrm{mM}$ IPTG and bacteria were incubated for 16 hours at $16^{\circ} \mathrm{C}$. Bacteria were pelleted at $6,000 \mathrm{~g}$ for $15 \mathrm{~min}$ and the pellet was resuspended in $120 \mathrm{ml}$ lysis buffer (20 mM HEPES-KOH pH 8, $400 \mathrm{mM} \mathrm{NaCl}, 0.5 \%$ NP40, 10\% glycerol, 30 $\mathrm{mM}$ imidazole, $1 \mathrm{mM}$ PMSF, $5 \mathrm{mM}$ beta-mercaptoethanol). The suspension was sonicated on ice at $70 \%$ amplitude with $15 \mathrm{sec}$ ON and $15 \mathrm{sec}$ OFF for 8 min total sonication time (Branson Sonifier). Insoluble material was removed by centrifugation for $25 \mathrm{~min}$ at 25,000g. In the meantime, $5 \mathrm{ml}$ packed Ni-NTA resin (Qiagen) were equilibrated in lysis buffer. The beads were added to the clarified lysate and incubated under rotation for $30 \mathrm{~min}$ at $4^{\circ} \mathrm{C}$. The bead suspension was added to a gravity flow column and the flow through was collected. Beads were washed with 40 ml lysis buffer, $120 \mathrm{ml}$ wash buffer (20 mM HEPES-KOH pH8, $1 \mathrm{M} \mathrm{NaCl}, 0.5 \%$ NP40, 10\% glycerol, $30 \mathrm{mM}$ imidazole), and $80 \mathrm{ml}$ lysis buffer. Proteins were eluted twice with $30 \mathrm{ml}$ elution buffer (20 mM TRIS-HCl pH 7.5, 300 mM NaCl, 300 mM imidazole). Two HiTrap Heparin $5 \mathrm{ml}$ columns (GE Healthcare) were installed in tandem on an Äkta chromatography system (GE Healthcare) and eliquibrated with 10 column volumes of HIEX buffer A (20 mM TRIS- $\mathrm{HCl} \mathrm{pH} 7.5,300 \mathrm{mM} \mathrm{NaCl}$ ). The Ni-NTA eluate was applied and the columns and washed with 10 column volumes of HIEX buffer $A$. Bound proteins were eluted into fractions with a linear salt gradient of HIEX buffer $A$ and HIEX buffer $\mathrm{B}$ (20 mM TRIS-HCl pH 7.5, $2 \mathrm{M} \mathrm{NaCl}$ ). Fractions were analysed by SDS-PAGE and the desired ones were pooled. After concentration (Amicon Ultra-15 $10 \mathrm{kD}$ Spin Filters, EMD Millipore) proteins were applied to a Superdex 75 increase 10/300 GI column and eluted with the final storage buffer (HEPES-KOH pH 7.5, $250 \mathrm{mM} \mathrm{KCl}, 1$ $\mathrm{mM}$ TCEP). Fractions were analysed by SDS-PAGE and the desired ones were pooled, concentrated, and stored at $-80^{\circ} \mathrm{C}$.

ORF9 purifications for phase separation assay (Figure S6C)

Recombinant ORF9 was purified using a protocol previously optimised for expression in MDG and M9ZB media (Zhou et al., 2019). Briefly, BL21-RIL DE3 E. coli (Agilent) were transformed with pET-6xHis-SUMO bacterial expression vectors for full-length ORF9 or C-terminally truncated ORF9 1-244 (ORF9-N). A starter culture of $30 \mathrm{ml}$ grown in MDG-medium was used to inoculate 2x $1 \mathrm{~L}$ M9ZB-media cultures. Cultures were grown to an $\mathrm{OD}$ of $\sim 2.5$, chilled to $16^{\circ} \mathrm{C}$, and protein expression was induced with $0.5 \mathrm{mM}$ IPTG before incubation at $16^{\circ} \mathrm{C}$ for $\sim 16$ hours. Pelleted bacteria were lysed in lysis buffer (20 mM HEPES-KOH pH 7.5, $400 \mathrm{mM} \mathrm{NaCl}, 10 \%$ glycerol, $30 \mathrm{mM}$ imidazole, $1 \mathrm{mM}$ DTT) by sonication. Insoluble material was removed by centrifugation and 6xHis-tagged protein was purified using Ni-NTA (Qiagen) and gravity chromatography. Proteins were eluted with $30 \mathrm{ml}$ elution buffer $(20 \mathrm{mM}$ HEPES-KOH $\mathrm{pH} 7.5,400 \mathrm{mM} \mathrm{NaCl}, 10 \%$ glycerol, $300 \mathrm{mM}$ imidazole, 1mM DTT), dialysed against low salt buffer $(150 \mathrm{mM} \mathrm{NaCl})$ overnight in the presence of $250 \mu \mathrm{g}$ hSENP2 protease for cleave of the 6xHis-SUMO tag. Cleaved protein was further purified by HiTrap SP ion-exchange for full length ORF9 and a combination of HiTrap Q ion-exchange and Superdex 75 size-exclusion chromatography for ORF9 1-244. Final purified fractions were pooled, concentrated and flash-frozen in liquid nitrogen for storage at $-80^{\circ} \mathrm{C}$.

cGAS

Recombinant human cGAS protein used for EMSA and cell-free cGAS activity assays was purified as described previously (Zhou et al., 2019). 
1047

1048

1049

1050

1051

1052

1053

1054

1055

1056

1057

1058

1059

1060

1061

1062

1063

1064

1065

1066

1067

1068

1069

1070

1071

1072

1073

1074

1075

1076

1077

1078

1079

1080

1081

1082

1083

1084

1085

1086

1087

1088

1089

1090

1091

\section{Immunoblotting}

Protein concentrations in lysates were determined using Pierce BCA Protein Assay Kit (Thermo Scientific) and equalised by dilution of samples with lysis buffer. Subsequently, 4x NuPAGE LDS Sample Buffer (Invitrogen) was added and samples were incubated at $95^{\circ} \mathrm{C}$ for $10 \mathrm{~min}$. Samples were run on NuPAGE Novex $4-12 \%$ BisTRIS gels (Invitrogen) using NuPage MOPS-SDS running buffer (Invitrogen). Proteins were subsequently blotted onto PROTRAN Pure nitrocellulose membrane (PerkinElmer) using transfer buffer (25mM TRIS, 192mM glycine). Membranes were blocked with 5\% skim milk powder (Sigma-Aldrich) in TBS containing 0.1\% Tween-20 (5\% milk TBS-T) for 1 hour at room temperature and were then incubated with primary antibodies in $5 \%$ milk TBS-T overnight at $4^{\circ} \mathrm{C}$. Primary antibodies that bind to phosphorylated residues were diluted in 5\% BSA in TBS-T. Membranes were washed thrice with TBS-T and incubated with HRP-coupled secondary antibodies in 5\% milk TBS-T for 1 hour at room temperature. After three further washes with TBS-T, proteins were detected using Western Lightning Plus-ECL (PerkinElmer) and the iBright FL1000 Imaging System (Thermo Fisher) or Amersham Hyperfilm MP (GE Healthcare). If needed, antibodies were stripped from the membrane with stripping buffer (200mM glycine, $0.1 \%$ SDS, $1 \%$ Tween-20, $\mathrm{pH} 2.2$ ) for $20 \mathrm{~min}$ at room temperature. Membranes were washed with TBS-T, blocked as described above and re-probed.

\section{$R T-q P C R$}

Cells were lysed in RLT Plus buffer and RNA was extracted using RNeasy Plus Mini Kit (Qiagen). The RNA was reverse transcribed using SuperScript IV Reverse Transcriptase (Invitrogen) and oligo-dT primers (Invitrogen). The qPCR reaction containing TaqMan Universal PCR Master Mix (Applied Biosystems) and TaqMan Primer/Probes was run on QuantStudio 7 Flex Real-Time PCR System (Thermo Fisher) with standard settings. Gene expression was analysed with the Ct method using GAPDH expression for normalisation. Taqman primer/probes used were: GAPDH (Hs02758991_g1), IFNB1 (Hs02621180_s1), IFI44 (Hs00951349_m1), and IFNL1 (Hs00601677_g1). RT-qPCR for VZV transcripts was performed with SYBR GreenER master mix (Invitrogen) using primers pairs indicated in Table S3.

\section{Cell-free cGAS activity assay}

Cell-free cGAS activity assays were performed according to a previously described procedure (Kranzusch et al., 2013). Full-length recombinant human cGAS protein (1 uM) and VACV70mer DNA (1 uM) were incubated in $20 \mathrm{ul}$ reaction buffer (50 mM TRIS-HCl $\mathrm{pH}$ 7.5, $10 \mathrm{mM} \mathrm{Mg}(\mathrm{OAc}) 2,10 \mathrm{mM} \mathrm{KCl}, 1 \mathrm{mM}$ DTT, $25 \mu \mathrm{M}$ ATP, $25 \mu \mathrm{M}$ GTP, $1 \mu \mathrm{Ci}$ a32P-ATP (PerkinElmer)) for $90 \mathrm{~min}$ at $37^{\circ} \mathrm{C}$. Reactions were terminated for $5 \mathrm{~min}$ at $95^{\circ} \mathrm{C}$. Leftover ATP was converted to inorganic phosphate by addition of $10 \mathrm{U}$ calfintestinal phosphatase and incubation for $20 \mathrm{~min}$ at $37^{\circ} .2 \mu \mathrm{l}$ of each reaction were 
1092

1093

1094

1095

1096

1097

1098

1099

1100

1101

1102

1103

1104

1105

1106

1107

1108

1109

1110

1111

1112

1113

1114

1115

1116

1117

1118

1119

1120

1121

1122

1123

1124

1125

1126

1127

1128

1129

1130

1131

1132

1133

1134

1135

spotted onto a PEI Celluose $\mathrm{F}$ thin-layer chromatography plate (EMD Millipore) and run in $1.5 \mathrm{M} \mathrm{KH}_{2} \mathrm{PO}_{4}$. Plates were dried at room temperature and exposed to a phosphorscreen overnight. Screens were imaged using a Typhoon FLA 9500 imager (GE Healthcare). Signal intensities were quantified using Fiji 2.0.0 software (Schindelin et al., 2012) and normalized to the average signal in conditions with BSA.

\section{Phase separation assays}

In vitro phase separation was performed as previously described (Du and Chen, 2018; Zhou et al., 2021). Briefly, human cGAS was labelled with AlexaFluor-488 (AF488) carboxylic acid (succinimidyl ester) (Thermo Fisher Scientific) according to manufacturer's manuals using a molar ratio of $1: 10$ at $4^{\circ} \mathrm{C}$ for $4 \mathrm{~h}$. Excess free dye was removed by dialysis against buffer (20 mM HEPES-KOH pH 7.5, $250 \mathrm{mM} \mathrm{KCl,} 1 \mathrm{mM}$ DTT) at $4^{\circ} \mathrm{C}$ overnight and then human cGAS was further purified on a PD-10 desalting column (GE Healthcare) eluted with storage buffer (20 mM HEPES-KOH pH 7.5, 250 $\mathrm{mM} \mathrm{KCl}, 1 \mathrm{mM}$ TCEP) as previously described (Zhou et al., 2021).

To induce phase separation, human cGAS, ORF9, or ORF9 truncation (10 $\mu \mathrm{M}$ each) was incubated with $100 \mathrm{bp}$ dsDNA (10 $\mu \mathrm{M}$, containing $1 \mu \mathrm{M}$ Cy3-labelled DNA) in the presence of various salt concentrations at $25^{\circ} \mathrm{C}$ in a total reaction volume of $20 \mu \mathrm{L}$. The details of proteins, nucleic acids, and salt concentrations are provided in figure legends. Reactions were placed in 384-well non-binding microplates (Greiner Bio-One) and incubated at $25^{\circ} \mathrm{C}$ for 30 min prior to imaging to allow condensates to settle. Fluorescence microscopy images were acquired at $25^{\circ} \mathrm{C}$ using a Leica TCS SP5 X (Leica Microsystems) mounted on an inverted microscope (DMI6000; Leica Microsystems) with an oil immersion $63 \times$ /numerical aperture 1.4 objective lens (HCX PL APO; Leica Microsystems). Labelled proteins were detected with excitation at 488 $\mathrm{nm}$ (emission at 500-530 nm) and DNA was detected with excitation at $550 \mathrm{~nm}$ (emission at 560-590 nm). Microscopy images were processed with FIJl (schindelin et al., 2012), and contrast adjusted with a uniform threshold setup for each enzyme.

\section{Protein sequence analysis}

The HSV-1 VP22 (PDB: 4XAL) and MHV68 ORF52 (PDB: 2OA5) crystal structures were used to graphically represent secondary structure features. For VZV ORF9 (UniProt accession Q4JQW6) and KSHV ORF52 (UniProt accession F5HBL8), structural features and sequence disorder was predicted by the PROFphd secondary structure prediction algorithm through submission of protein sequences to the predictprotein.org web interface (Yachdav et al., 2014).

\section{Data Analysis and Software}

Data were analysed using Excel for Mac (Microsoft) and GraphPad Prism 8 (GraphPad Software). SnapGene (GSL Biotech) and ApE (M. Wayne Davis, The University of Utah) were utilised for DNA sequence analysis to assist cloning. Statistical analysis is detailed in the figure legends and was performed using GraphPad Prism 8. Graphs 
1136 and figures were created using GraphPad Prism 8 and Adobe Illustrator CC (Adobe 1137 Systems). Immunoblot images were processed using web-based iBright Image 1138 Analysis software (Thermo Fisher). Flow cytometry data was analysed using FlowJo 1139 (FlowJo, LLC). Fiji 2.0.0 software was used to process confocal microscopy images 1140 (Schindelin et al., 2012). 
1141

1142

1143

1144

1145

1146

1147

1148

1149

1150

1151

1152

1153

1154

1155

1156

1157

1158

1159

1160

1161

1162

1163

1164

1165

1166

1167

1168

1169

1170

1171

1172

1173

1174

1175

1176

1177

1178

1179

1180

\section{REFERENCES}

Abendroth, A., Morrow, G., Cunningham, A.L., and Slobedman, B. (2001). Varicellazoster virus infection of human dendritic cells and transmission to T cells: implications for virus dissemination in the host. J Virol 75, 6183-6192.

Ablasser, A., and Chen, Z.J. (2019). cGAS in action: Expanding roles in immunity and inflammation. Science 363, eaat8657.

Almine, J.F., O'Hare, C.A., Dunphy, G., Haga, I.R., Naik, R.J., Atrih, A., Connolly, D.J., Taylor, J., Kelsall, I.R., Bowie, A.G., et al. (2017). IFI16 and cGAS cooperate in the activation of STING during DNA sensing in human keratinocytes. Nat Commun 8 , 14392.

Andreeva, L., Hiller, B., Kostrewa, D., Lassig, C., de Oliveira Mann, C.C., Jan Drexler, D., Maiser, A., Gaidt, M., Leonhardt, H., Hornung, V., et al. (2017). cGAS senses long and HMGB/TFAM-bound U-turn DNA by forming protein-DNA ladders. Nature 549, 394-398.

Arvin, A.M., and Gilden, D. (2013). Varicella-Zoster Virus. In Virology, B.N. Fields, D.M. Knipe, and P.M. Howley, eds. (Philadelphia: Wolters Kluwer Health/Lippincott Williams \& Wilkins), pp. 2015-2057.

Arvin, A.M., Koropchak, C.M., Williams, B.R.G., Grumet, F.C., and Foung, S.K.H. (1986). Early Immune Response in Healthy and Immunocompromised Subjects with Primary Varicella-Zoster Virus Infection. The Journal of Infectious Diseases 154, 422429.

Barrat, F.J., Elkon, K.B., and Fitzgerald, K.A. (2016). Importance of Nucleic Acid Recognition in Inflammation and Autoimmunity. Annu Rev Med 67, 323-336.

Bridgeman, A., Maelfait, J., Davenne, T., Partridge, T., Peng, Y., Mayer, A., Dong, T., Kaever, V., Borrow, P., and Rehwinkel, J. (2015). Viruses transfer the antiviral second messenger cGAMP between cells. Science 349, 1228-1232.

Brinkman, E.K., Chen, T., Amendola, M., and van Steensel, B. (2014). Easy quantitative assessment of genome editing by sequence trace decomposition. Nucleic Acids Res 42, e168-e168.

Brito, A.F., and Pinney, J.W. (2018). Time-calibrated tree reconciliations reveal frequent losses, intrahost speciations, and host switches in the evolution of herpesviruses. Biorxiv, 418111.

Brubaker, S.W., Bonham, K.S., Zanoni, I., and Kagan, J.C. (2015). Innate immune pattern recognition: a cell biological perspective. Annu Rev Immunol 33, 257-290.

Buchan, D.W.A., and Jones, D.T. (2019). The PSIPRED Protein Analysis Workbench: 20 years on. Nucleic Acids Res 47, W402-W407.

Campbell, T.M., McSharry, B.P., Steain, M., Ashhurst, T.M., Slobedman, B., and Abendroth, A. (2018). Varicella zoster virus productively infects human natural killer cells and manipulates phenotype. PLoS Pathog 14, e1006999. 
Carter-Timofte, M.E., Paludan, S.R., and Mogensen, T.H. (2018). RNA Polymerase III as a Gatekeeper to Prevent Severe VZV Infections. Trends in Molecular Medicine 24, 904-915.

Caunt, A.E., and Taylor-Robinson, D. (2009). Cell-free varicella-zoster virus in tissue culture. Epidemiol Infect 62, 413-424.

Che, X., Oliver, S.L., Reichelt, M., Sommer, M.H., Haas, J., Rovis, T.L., and Arvin, A.M. (2013). ORF11 Protein Interacts with the ORF9 Essential Tegument Protein in Varicella-Zoster Virus Infection. J Virol 87, 5106-5117.

Che, X., Reichelt, M., Sommer, M.H., Rajamani, J., Zerboni, L., and Arvin, A.M. (2008). Functions of the ORF9-to-ORF12 Gene Cluster in Varicella-Zoster Virus Replication and in the Pathogenesis of Skin Infection. J Virol 82, 5825-5834.

Chen, J.J., Zhu, Z., Gershon, A.A., and Gershon, M.D. (2004). Mannose 6Phosphate Receptor Dependence of Varicella Zoster Virus Infection In Vitro and in the Epidermis during Varicella and Zoster. Cell 119, 915-926.

Cohen, J.I., and Seidel, K.E. (1993). Generation of varicella-zoster virus (VZV) and viral mutants from cosmid DNAs: VZV thymidylate synthetase is not essential for replication in vitro. Proc Natl Acad Sci USA 90, 7376-7380.

Davison, A.J. (2002). Evolution of the herpesviruses. Vet Microbiol 86, 69-88.

Drayman, N., Patel, P., Vistain, L., and Tay, S. (2019). HSV-1 single-cell analysis reveals the activation of anti-viral and developmental programs in distinct subpopulations. Elife 8, 2503.

Du, M., and Chen, Z.J. (2018). DNA-induced liquid phase condensation of cGAS activates innate immune signaling. Science 361, 704-709.

Freundt, E.C., Drappier, M., and Michiels, T. (2018). Innate Immune Detection of Cardioviruses and Viral Disruption of Interferon Signaling. Front Microbiol 9, 867.

Gerada, C., Campbell, T.M., Kennedy, J.J., McSharry, B.P., Steain, M., Slobedman, B., and Abendroth, A. (2020). Manipulation of the Innate Immune Response by Varicella Zoster Virus. Front Immunol 11, 1.

Haberthur, K., and Messaoudi, I. (2013). Animal Models of Varicella Zoster Virus Infection. Pathogens 2, 364-382.

Harding, S.M., Benci, J.L., Irianto, J., Discher, D.E., Minn, A.J., and Greenberg, R.A. (2017). Mitotic progression following DNA damage enables pattern recognition within micronuclei. Nature 548, 466-470.

Hartmann, G. (2017). Nucleic Acid Immunity. Adv Immunol 133, 121-169.

Hertzog, J., Dias Junior, A.G., Rigby, R.E., Donald, C.L., Mayer, A., Sezgin, E., Song, C., Jin, B., Hublitz, P., Eggeling, C., et al. (2018). Infection with a Brazilian isolate of Zika virus generates RIG-I stimulatory RNA and the viral NS5 protein blocks type I IFN induction and signaling. Eur J Immunol 48, 1120-1136. 
Hew, K., Dahlroth, S.L., Pan, L.X., Cornvik, T., and Nordlund, P. (2015). VP22 core domain from Herpes simplex virus 1 reveals a surprising structural conservation in both the Alpha- and Gammaherpesvirinae subfamilies. J Gen Virol 96, 1436-1445.

Holm, C.K., Rahbek, S.H., Gad, H.H., Bak, R.O., Jakobsen, M.R., Jiang, Z., Hansen, A.L., Jensen, S.K., Sun, C., Thomsen, M.K., et al. (2016). Influenza A virus targets a cGAS-independent STING pathway that controls enveloped RNA viruses. Nat Commun 7, 10680.

Hopfner, K.P., and Hornung, V. (2020). Molecular mechanisms and cellular functions of cGAS-STING signalling. Nat Rev Mol Cell Biol 21, 501-521.

Horan, K.A., Hansen, K., Jakobsen, M.R., Holm, C.K., Soby, S., Unterholzner, L., Thompson, M., West, J.A., Iversen, M.B., Rasmussen, S.B., et al. (2013).

Proteasomal degradation of herpes simplex virus capsids in macrophages releases DNA to the cytosol for recognition by DNA sensors. J Immunol 190, 2311-2319.

Hu, S., Sun, H., Yin, L., Li, J., Mei, S., Xu, F., Wu, C., Liu, X., Zhao, F., Zhang, D., et al. (2019). PKR-dependent cytosolic cGAS foci are necessary for intracellular DNA sensing. Science Signaling 12, eaav7934.

Huang, J., You, H., Su, C., Li, Y., Chen, S., and Zheng, C. (2018). Herpes Simplex Virus 1 Tegument Protein VP22 Abrogates cGAS/STING-Mediated Antiviral Innate Immunity. J Virol 92, 783.

Kennedy, J.J., Steain, M., Slobedman, B., and Abendroth, A. (2019). Infection and Functional Modulation of Human Monocytes and Macrophages by Varicella-Zoster Virus. J Virol 93, 10980.

Kim, J.-A., Park, S.-K., Seo, S.-w., Lee, C.H., and Shin, O.S. (2017). STING Is Involved in Antiviral Immune Response against VZV Infection via the Induction of Type I and III IFN Pathways. Journal of Investigative Dermatology 137, 2101-2109.

Kranzusch, P.J., Lee, A.S., Berger, J.M., and Doudna, J.A. (2013). Structure of human cGAS reveals a conserved family of second-messenger enzymes in innate immunity. Cell Rep 3, 1362-1368.

Kranzusch, P.J., Wilson, S.C., Lee, A.S., Berger, J.M., Doudna, J.A., and Vance, R.E. (2015). Ancient Origin of cGAS-STING Reveals Mechanism of Universal 2',3' cGAMP Signaling. Mol Cell 59, 891-903.

Ku, C.-C., Chang, Y.-H., Chien, Y., and Lee, T.-L. (2016). Type I interferon inhibits varicella-zoster virus replication by interfering with the dynamic interaction between mediator and IE62 within replication compartments. Cell Biosci 6, 519.

Lebrun, M., Lambert, J., Riva, L., Thelen, N., Rambout, X., Blondeau, C., Thiry, M., Snoeck, R., Twizere, J.-C., Dequiedt, F., et al. (2018). Varicella-Zoster Virus ORF9p Binding to Cellular Adaptor Protein Complex 1 Is Important for Viral Infectivity. J Virol 92, 222. 
1259 Is an Abundant Tegument Protein and Is Required for Production of Infectious

1260 Progeny Viruses. J Virol 90, 5329-5342.

1261 Li, X., Shu, C., Yi, G., Chaton, C.T., Shelton, C.L., Diao, J., Zuo, X., Kao, C.C., Herr, 1262 A.B., and Li, P. (2013). Cyclic GMP-AMP synthase is activated by double-stranded 1263 DNA-induced oligomerization. Immunity 39, 1019-1031.

1264 Luecke, S., Holleufer, A., Christensen, M.H., Jonsson, K.L., Boni, G.A., Sorensen, 1265 L.K., Johannsen, M., Jakobsen, M.R., Hartmann, R., and Paludan, S.R. (2017). 1266 cGAS is activated by DNA in a length-dependent manner. EMBO Rep 18, 170712671715.

Lum, K.K., Song, B., Federspiel, J.D., Diner, B.A., Howard, T., and Cristea, I.M. (2018). Interactome and Proteome Dynamics Uncover Immune Modulatory Associations of the Pathogen Sensing Factor cGAS. Cell Syst 7, 627-642 e626.

Mackenzie, K.J., Carroll, P., Martin, C.A., Murina, O., Fluteau, A., Simpson, D.J., Olova, N., Sutcliffe, H., Rainger, J.K., Leitch, A., et al. (2017). cGAS surveillance of micronuclei links genome instability to innate immunity. Nature 548, 461-465.

Maelfait, J., Liverpool, L., and Rehwinkel, J. (2020). Nucleic Acid Sensors and Programmed Cell Death. J Mol Biol 432, 552-568.

Mainka, C., Fuss, B., Geiger, H., Höfelmayr, H., and Wolff, M.H. (1998). Molecular Phylogeny and Evolutionary Timescale for the Family of Mammalian Herpesviruses. Journal of Molecular Biology 247, 443-458.

1282 McNab, F., Mayer-Barber, K., Sher, A., Wack, A., and O'Garra, A. (2015). Type I interferons in infectious disease. Nat Rev Immunol 15, 87-103. infection facilitates VZV glycoprotein E trafficking to the membrane surface of melanoma cells. J Med Virol 70, S56-S58.

1287 Morrow, G., Slobedman, B., Cunningham, A.L., and Abendroth, A. (2003). VaricellaZoster Virus Productively Infects Mature Dendritic Cells and Alters Their Immune Function. J Virol 77, 4950-4959.

1290 Nagel, M.A., and Gilden, D. (2014). Neurological complications of varicella zoster virus reactivation. Current Opinion in Neurology 27, 356-360. (2011). Varicella-Zoster Virus Infection Triggers Formation of an Interleukin-1 $\beta$ (IL1ß)-processing Inflammasome Complex. J Biol Chem 286, 17921-17933. 
Rehwinkel, J., Tan, C.P., Goubau, D., Schulz, O., Pichlmair, A., Bier, K., Robb, N., Vreede, F., Barclay, W., Fodor, E., et al. (2010). RIG-I Detects Viral Genomic RNA during Negative-Strand RNA Virus Infection. Cell 140, 397-408.

Riva, L., Thiry, M., Bontems, S., Joris, A., Piette, J., Lebrun, M., and Sadzot-Delvaux, C. (2013). ORF9p Phosphorylation by ORF47p Is Crucial for the Formation and Egress of Varicella-Zoster Virus Viral Particles. J Virol 87, 2868-2881.

Riva, L., Thiry, M., Lebrun, M., L'Homme, L., Piette, J., and Sadzot-Delvaux, C. (2015). Deletion of the ORF9p Acidic Cluster Impairs the Nuclear Egress of VaricellaZoster Virus Capsids. J Virol 89, 2436-2441.

Schindelin, J., Arganda-Carreras, I., Frise, E., Kaynig, V., Longair, M., Pietzsch, T., Preibisch, S., Rueden, C., Saalfeld, S., Schmid, B., et al. (2012). Fiji: an open-source platform for biological-image analysis. Nat Methods 9, 676-682.

Schoggins, J.W. (2019). Interferon-Stimulated Genes: What Do They All Do? Annu Rev Virol 6, 567-584.

Sen, N., Sommer, M., Che, X., White, K., Ruyechan, W.T., and Arvin, A.M. (2010). Varicella-Zoster Virus Immediate-Early Protein 62 Blocks Interferon Regulatory Factor 3 (IRF3) Phosphorylation at Key Serine Residues: a Novel Mechanism of IRF3 Inhibition among Herpesviruses. J Virol 84, 9240-9253.

Shakya, A.K., O'Callaghan, D.J., and Kim, S.K. (2019). Interferon Gamma Inhibits Varicella-Zoster Virus Replication in a Cell Line-Dependent Manner. J Virol 93, 3904.

Smith, G.L., Talbot-Cooper, C., and Lu, Y. (2018). Chapter Fourteen - How Does Vaccinia Virus Interfere With Interferon? In Advances in Virus Research, M. Kielian, T.C. Mettenleiter, and M.J. Roossinck, eds. (Academic Press), pp. 355-378.

Stempel, M., Chan, B., and Brinkmann, M.M. (2019). Coevolution pays off: Herpesviruses have the license to escape the DNA sensing pathway. Med Microbiol Immunol 208, 495-512.

Sun, L., Wu, J., Du, F., Chen, X., and Chen, Z.J. (2013). Cyclic GMP-AMP synthase is a cytosolic DNA sensor that activates the type I interferon pathway. Science 339, 786-791.

Tischer, B.K., Kaufer, B.B., Sommer, M., Wussow, F., Arvin, A.M., and Osterrieder, N. (2007). A Self-Excisable Infectious Bacterial Artificial Chromosome Clone of Varicella-Zoster Virus Allows Analysis of the Essential Tegument Protein Encoded by ORF9. J Virol 81, 13200-13208.

Torigo, S., Ihara, T., and Kamiya, H. (2000). IL-12, IFN-gamma, and TNF-alpha released from mononuclear cells inhibit the spread of varicella-zoster virus at an early stage of varicella. Microbiol Immunol 44, 1027-1031.

Uetz, P., Dong, Y.-A., Zeretzke, C., Atzler, C., Baiker, A., Berger, B., Rajagopala, S.V., Roupelieva, M., Rose, D., Fossum, E., et al. (2006). Herpesviral Protein Networks and Their Interaction with the Human Proteome. Science 311, 239. 
Unterholzner, L., Keating, S.E., Baran, M., Horan, K.A., Jensen, S.B., Sharma, S., Sirois, C.M., Jin, T., Latz, E., Xiao, T.S., et al. (2010). IFI16 is an innate immune sensor for intracellular DNA. Nat Immunol 11, 997-1004.

Wang, J.P., Kurt-Jones, E.A., Shin, O.S., Manchak, M.D., Levin, M.J., and Finberg, R.W. (2005). Varicella-Zoster Virus Activates Inflammatory Cytokines in Human Monocytes and Macrophages via Toll-Like Receptor 2. J Virol 79, 12658-12666.

West, A.P., Khoury-Hanold, W., Staron, M., Tal, M.C., Pineda, C.M., Lang, S.M., Bestwick, M., Duguay, B.A., Raimundo, N., MacDuff, D.A., et al. (2015).

Mitochondrial DNA stress primes the antiviral innate immune response. Nature 520, 553-557.

WHO (2014). Varicella and herpes zoster vaccines: WHO position paper, June 2014. Weekly epidemiological record 25, 265-288.

Wu, J., Sun, L., Chen, X., Du, F., Shi, H., Chen, C., and Chen, Z.J. (2013). Cyclic GMP-AMP is an endogenous second messenger in innate immune signaling by cytosolic DNA. Science 339, 826-830.

Wu, J.J., Li, W., Shao, Y., Avey, D., Fu, B., Gillen, J., Hand, T., Ma, S., Liu, X., Miley, W., et al. (2015). Inhibition of cGAS DNA Sensing by a Herpesvirus Virion Protein. Cell Host Microbe 18, 333-344.

Wu, Y., Li, Q., and Chen, X.-Z. (2007). Detecting protein-protein interactions by far western blotting. Nat Protoc 2, 3278-3284.

Xie, W., Lama, L., Adura, C., Tomita, D., Glickman, J.F., Tuschl, T., and Patel, D.J. (2019). Human cGAS catalytic domain has an additional DNA-binding interface that enhances enzymatic activity and liquid-phase condensation. Proc Natl Acad Sci U S A 116, 11946-11955.

Yachdav, G., Kloppmann, E., Kajan, L., Hecht, M., Goldberg, T., Hamp, T., Honigschmid, P., Schafferhans, A., Roos, M., Bernhofer, M., et al. (2014). PredictProtein-an open resource for online prediction of protein structural and functional features. Nucleic Acids Research 42, W337-W343.

Yoneyama, M., Kikuchi, M., Natsukawa, T., Shinobu, N., Imaizumi, T., Miyagishi, M., Taira, K., Akira, S., and Fujita, T. (2004). The RNA helicase RIG-I has an essential function in double-stranded RNA-induced innate antiviral responses. Nat Immunol 5, 730-737.

Yu, H.-R., Huang, H.-C., Kuo, H.-C., Sheen, J.-M., Ou, C.-Y., Hsu, T.-Y., and Yang, K.D. (2011). IFN- $\alpha$ production by human mononuclear cells infected with varicellazoster virus through TLR9-dependent and -independent pathways. Cell Mol Immunol $8,181-188$.

Zerboni, L., Sen, N., Oliver, S.L., and Arvin, A.M. (2014). Molecular mechanisms of varicella zoster virus pathogenesis. Nat Rev Microbiol 12, 197-210.

Zhang, X., Wu, J., Du, F., Xu, H., Sun, L., Chen, Z., Brautigam, C.A., Zhang, X., and Chen, Z.J. (2014). The cytosolic DNA sensor cGAS forms an oligomeric complex 
1376 with DNA and undergoes switch-like conformational changes in the activation loop.

1377 Cell Rep 6, 421-430.

1378 Zhou, W., Mohr, L., Maciejowski, J., and Kranzusch, P.J. (2021). cGAS phase

1379 separation inhibits TREX1-mediated DNA degradation and enhances cytosolic DNA 1380 sensing. Mol Cell 81, 739-755 e737.

1381 Zhou, W., Whiteley, A.T., de Oliveira Mann, C.C., Morehouse, B.R., Nowak, R.P., 1382 Fischer, E.S., Gray, N.S., Mekalanos, J.J., and Kranzusch, P.J. (2018). Structure of 1383 the Human cGAS-DNA Complex Reveals Enhanced Control of Immune Surveillance. 1384 Cell 174, 300-311 e311.

1385 Zhou, W., Whiteley, A.T., and Kranzusch, P.J. (2019). Analysis of human cGAS 1386 activity and structure. Methods Enzymol 625, 13-40.

1387 Zhu, H., Zheng, C., Xing, J., Wang, S., Li, S., Lin, R., and Mossman, K.L. (2011).

1388 Varicella-Zoster Virus Immediate-Early Protein ORF61 Abrogates the IRF3-Mediated 1389 Innate Immune Response through Degradation of Activated IRF3. J Virol 85, 11079139011089. 